Fachbereich 5

Wirtschaftswissenschaften, Wirtschaftsinformatik und Wirtschaftsrecht

Volkswirtschaftliche Diskussionsbeiträge

Discussion Papers in Economics

No. 136-09

August 2009

Thomas Eichner · Rüdiger Pethig

Carbon leakage, the green paradox and perfect future markets 


\section{Universität Siegen}

Fachbereich 5

Wirtschaftswissenschaften, Wirtschaftsinformatik und Wirtschaftsrecht

Fachgebiet Volkswirtschaftslehre

Hölderlinstraße 3

D-57068 Siegen

Germany

http://www.uni-siegen.de/fb5/vwl/

ISSN 1869-0211

Available for free from the University of Siegen website at http://www.uni-siegen.de/fb5/vwl/research/diskussionsbeitraege/

Discussion Papers in Economics of the University of Siegen are indexed in RePEc and can be downloaded free of charge from the following website:

http://ideas.repec.org/s/sie/siegen.html 


\title{
Carbon leakage, the green paradox and perfect future markets*
}

\author{
Thomas Eichner \\ Department of Economics, University of Bielefeld \\ Rüdiger Pethig \\ Department of Economics, University of Siegen
}

\begin{abstract}
Policies of lowering carbon demand may aggravate rather than alleviate climate change (green paradox). In a two-period three-country general equilibrium model with finite endowment of fossil fuel one country enforces an emissions cap in the first or second period. When that cap is tightened the extent of carbon leakage depends on the interaction of various parameters and elasticities. Conditions for the green paradox are specified. All determinants of carbon leakage resulting from tightening the first-period cap work in opposite direction when the second-period cap is tightened. Tightening the second-period cap does not necessarily lead to the green paradox.

JEL classification: H22, Q32, Q54

Key words: $\quad$ carbon leakage, green paradox, emissions cap
\end{abstract}

*Eichner: Department of Economics, University of Bielefeld, Universitätsstr. 25, 33615 Bielefeld, Germany, phone: ++ 49 (0) 521106 4857, email: teichner@wiwi.uni-bielefeld.de; Pethig: Department of Economics, University of Siegen, Hölderlinstr. 3, 57068 Siegen, Germany, phone: ++ 49 (0) 2717403143 , email: pethig@vwl.wiwi.uni-siegen.de 


\section{The problem}

Growing scientific evidence (IPCC 2007) suggests that we cannot stabilize the world climate at safe levels unless we substantially slow down the world emissions of greenhouse gases during the next decades. A number of countries have already increased their efforts to curb emissions, notably the (Annex 1) countries that committed to emissions reductions in the Kyoto Protocol. Yet many small and large countries still refrain from taking (strong) action. That raises the question what the chances are of a subset of abating countries to bring down world emissions to safe levels.

We will address this issue by restricting our focus on carbon dioxide that is the most important greenhouse gas. Carbon dioxide emissions (emissions, for short) are generated almost in proportion to burning fossil energy resources (fossil fuel, for short) which are still the dominant source of energy consumption. Any national policy of curbing emissions is bound to raise domestic energy costs and thus enables firms in non-abating countries to expand. For that reason, the effort of abating countries will be offset to some extent by increasing emissions in non-abating countries. That phenomenon has come to be known as carbon leakage. Since it is the aggregate world emissions that determine the damage from climate change, the net emissions cutback by a group of abating countries is smaller than that group's gross emissions reductions. It is conceivable that the induced emissions increase in non-abating countries is equal to or even greater than the gross emissions reduction achieved by the group of abating countries. The extreme case in which demand-reducing measures of abating countries increase rather than reduce aggregate world emissions, as compared to their level in the absence of abatement efforts, is referred to as green paradox by Sinn (2008).

High rates of carbon leakage would cast serious doubt on the effectiveness of any subglobal abatement strategy as represented, e.g., by the Kyoto approach. Since it is unclear at present whether an effective post-Kyoto agreement will be reached over the next years mandating strong action for all major carbon emitting countries, it is important to have a good understanding of the key mechanisms underlying carbon leakage.

The bulk of research on carbon leakage has been carried out in (large-scale) CGE analyses. According to Burniaux and Martins (2000) the estimates of such models range from leakage rates of $20 \%$ to lower bound estimates of $2 \%$ to $5 \%$. The IPCC (2007) estimates the leakage effect in about the same range for the climate policy based on the Kyoto Protocol. Burniaux and Martins (2000) conclude from their extensive sensitivity analysis (ibidem, p. 13) that "... carbon leakages are small for the range of parameters most frequently quoted in the literature ...", and they emphasize that this assessment strongly relies on the 
assumption that the supply of coal is fairly elastic over the medium term. Bohm (1993), Felder and Rutherford (1993), Hoel (1996), Paltsev (2001), Babiker (2005), Copeland and Taylor (2005), Ishikawa and Kiyono (2006), Gerlagh and Kuik (2007), Ishikawa and Okubo (2008), Marschinski et al. (2008) and others provide further informative insights into various channels and determinants of carbon leakage.

The prevailing view of relatively modest leakage rates is challenged by a line of research in the area of (intertemporal) theory of nonrenewable natural resources that takes as its point of departure an extraction path of fossil fuel that is suboptimally steep in laissezfaire e.g. because of the global warming externality (Sinn 1982; Sinclair 1994; Hoel and Kverndokk 1996; Farzin 1996; Rubio and Escriche 2001; Sinn 2008). In models that differ with respect to their assumptions on market power and strategic behavior the question is addressed as to what the potential is of various kinds of taxation to restore efficiency by flattening the extraction path. Under various qualifications a major though not undisputed result is that carbon taxes tend to have little impact on the time profile of extraction and that the extraction path is steepened if tax rates rise in time. Accordingly, Sinn's (2008, p. 360) verdict is that "... if suppliers feel threatened by a gradual greening of (demandreducing) policies in the Kyoto countries that would damage their future prices; they will extract their stocks more rapidly, thus accelerating global warming" (Sinn 2008, p. 360). From this perspective the prevailing view on the effectiveness of demand-reducing policies is flawed because the public and academic discourse (including the Stern Review 2006) has largely neglected the close link between the economics of global change and the economics of non-renewable resources and has therefore failed to account for the supply side of the problem in an appropriate way.

The supply-side literature aggregates all fossil-fuel consuming countries into a single country which amounts to presupposing full cooperation of all countries. Yet the very notion of carbon leakage as introduced above requires distinguishing abating and nonabating countries since carbon emissions leak from the former to the latter, after all. ${ }^{1}$ We are not aware of analytical studies that model intertemporal wealth maximizing resource supply and consider, at the same time, the leakage of carbon from the group of abating countries to non-abating countries.

The important insight of the supply-side literature is that due to the resource suppliers' non-myopic behavior the flow of emissions in the near future ('first period') which needs

\footnotetext{
${ }^{1}$ This is not to say that approaches focusing on global centralized emissions control policies are irrelevant for the topic of the present paper. A green paradox can certainly be said to occur when a global policy intending to flatten the extraction path results in steepening it. For the link between that literature and the present paper see also Section 4.0.
} 
to be reduced for climate stabilization is affected by demand reducing policies in the near future ('first-period') as well as by (credibly announced) demand reducing policies in the far future ('second period'). We take up that insight in the present paper which aims at examining the determinants of carbon leakage and the green paradox in a model that is stripped of complexities beyond the essentials of the problem. Our two-period general-equilibrium model focuses on the intertemporal use of fossil fuel as a non-renewable resource in finite supply and maps the prevailing real-world scenario in a stylized way as a three-country economy consisting of a fossil-fuel exporting country, an abating country and a non-abating country. The abating country represents the coalition of countries that have committed to observe binding national emissions caps à la Kyoto and the non-abating country stands for the rest of the world (except the fuel exporting countries) assumed to refrain from taking (strong) action to curb emissions. To keep a clear focus on leakage, we neither deal with capital formation and extraction costs nor with environmental damage from carbon emissions and optimal corrective policies.

In that general equilibrium framework we explore the conditions for carbon leakage and the green paradox. We investigate by means of comparative static analysis how much carbon leaks into the non-abating country when the abating country tightens its emissions cap and when the resource supplier follows a (simplified) Hotelling rule. We find that carbon leakage is unavoidable and we characterize the determinants of the green paradox which consist of an interplay of demand conditions, in particular the elasticity of intertemporal substitution in demand, and supply conditions, especially the price elasticities of fuel demand. We derive parameter constellations under which the green paradox occurs when the emissions constraint is tightened either in the first or in the second period. The proposition which ties the green paradox to the gradual greening of demand-reducing policies therefore does not receive unambiguous support from our analysis. ${ }^{2}$ When more countries join the coalition of abating countries, less carbon tends to leak into the non-abating countries. The incidence of reducing emissions (= tightening the emissions cap) either in the first or in the second period is shown to be mirror-symmetric: Essentially, parameter constellations under which the green paradox is avoided, when the first-period cap is tightened, tend to generate a green paradox, when the second-period cap is tightened, and vice versa.

The paper is organized as follows. Section 2 sets up the model. Section 3 investigates the determinants of carbon leakage and the green paradox when the abating country tightens its first-period emissions cap. Section 4 explores the effects of enlarging the group of abating countries. In Section 5 the same issues as in Section 3 are addressed for the case

\footnotetext{
${ }^{2}$ See also Ulph and Ulph (1994) who show in a different analytical framework that the optimal carbon tax need not necessarily be falling.
} 
that the abating country tightens its second-period emissions cap. Section 6 concludes.

\section{The model}

Consider a two-period model with three (groups of) countries $A, N$ and $F$, where $A$ is the abating country, $N$ is the non-abating country and $F$ is the fossil-fuel exporting country. Except for their carbon emissions control (see below) the economies of the countries $A$ and $N$ are alike. In period $t=1,2$ each country $i=A, N$ produces the output $x_{i t}^{s}$ of the consumption good $X$ with the input $e_{i t}$ of fossil fuel according to the increasing and strictly concave production function ${ }^{3}$

$$
x_{i t}^{s}=X^{i}\left(e_{i t}\right) \quad i=A, N .
$$

The countries $A$ and $N$ import all fossil fuel from country $F$ that is endowed with a stock of fossil fuel, $\bar{e}$. Country $F$ does not produce good $X$ but rather buys that good from the countries $A$ and $N$ paying for those imports with the revenues from exporting fossil fuel.

The representative consumer of country $i$ derives utility from consumption $x_{i 1}$ in period 1 and from $x_{i 2}$ in period 2 according to the intertemporal utility function

$$
u_{i}=U^{i}\left(x_{i 1}, x_{i 2}\right) \equiv U\left(x_{i 1}, x_{i 2}\right) \quad i=A, F, N,
$$

which is increasing in both arguments, quasi-concave and homothetic. ${ }^{4}$ The elasticity of intertemporal substitution (in consumption), defined as

$$
\sigma_{i}:=\frac{\mathrm{d}\left(\frac{x_{i 2}}{x_{i 1}}\right)}{\frac{x_{i 2}}{x_{i 1}}} \cdot \frac{\frac{U_{x_{i 1}}}{U_{x_{i 2}}}}{\mathrm{~d}\left(\frac{U_{x_{i 1}}}{U_{x_{i 2}}}\right)} \in[0, \infty[,
$$

is a property of the utility function that will turn out to play an important role in the subsequent analysis.

In each period, good $X$ and fossil fuel are traded on perfectly competitive world markets (comprising all three countries) at prices $p_{x t}$ and $p_{e t}$, respectively. For $t=1,2$ the market clearing conditions are

$$
\begin{aligned}
x_{A t}^{s}+x_{N t}^{s} & =x_{A t}+x_{N t}+x_{F t}, \\
e_{F t} & =e_{A t}+e_{N t},
\end{aligned}
$$

\footnotetext{
${ }^{3}$ In (1) the superscript $s$ indicates quantities supplied. Upper case letters denote functions and subscripts attached to them indicate first partial derivatives.

${ }^{4} \mathrm{~A}$ function is homothetic, if it can be written as an increasing transform of a linear homogeneous function. The class of homothetic functions encompases CES functions, Leontief functions, and isoelastic functions. Isoelastic utility functions (see (26) below) are often applied in empirical studies and, e.g., in the Stern review (2006).
} 
where $e_{F t}$ is the fossil fuel supply of country $F$ in period $t$. Obviously, the supplies $e_{F t}$ for $t=1,2$ need to satisfy the intertemporal constraint

$$
\bar{e}=e_{F 1}+e_{F 2}
$$

The countries $A$ and $N$ differ with respect to their carbon emissions regulation. We envisage an international agreement on reducing carbon emissions like the Kyoto protocol that does not encompass all countries in the world. Country $N$ represents the group of fuel-consuming countries that do not participate in the agreement and hence do not constrain their carbon emissions at all. ${ }^{5}$ Country $A$ then represents the group of participating countries. Denote by $\bar{e}_{A t}$ the emissions cap country $A$ imposes in period $t=1,2$. Country $A$ may cap its emissions either in both periods or in one of the periods only. At present there is already a group of countries capping their emissions. Therefore, we will restrict our focus on the scenarios (i) where a binding cap $\bar{e}_{A 1}$ exists but no cap in period 2, i.e.

$$
e_{A 1}=\bar{e}_{A 1} \text { and } e_{A 2} \text { unconstrained, }
$$

or (ii) where binding caps exist in both periods, i.e.

$$
e_{A 1}=\bar{e}_{A 1} \quad \text { and } \quad e_{A 2}=\bar{e}_{A 2}
$$

The caps can either be imposed directly, or through a tax-and-standard scheme or through an emissions trading system. Given the high level of abstraction of our model all these policies of implementing an emissions cap are equivalent. To simplify the exposition we refer to emissions trading only in the subsequent analysis assuming that the emissions permits are auctioned at some price $\pi_{t}, t=1,2$, that is determined endogenously.

Each country hosts a representative firm generating the profit

$$
\begin{aligned}
\Pi^{A} & :=\sum_{t}\left[p_{x t} X^{A}\left(e_{A t}\right)-\left(p_{e t}+\pi_{t}\right) e_{A t}\right] \\
\Pi^{N} & :=\sum_{t}\left[p_{x t} X^{N}\left(e_{N t}\right)-p_{e t} e_{N t}\right], \\
\Pi^{F} & :=\sum_{t} p_{e t} e_{F t},
\end{aligned}
$$

where $\pi_{2} \equiv 0$ if (6a) applies. In (7) - (9) we have not discounted the second-period profits, because in the absence of capital investment the market rate of interest is zero. Moreover,

\footnotetext{
${ }^{5}$ When climate is treated as a global public good, the business-as-usual scenario is commonly modeled as a Nash equilibrium where each country's emissions-reduction policy is the best reply to the other countries' abatement efforts. For the resultant "free-rider leakage" in such an approach see e.g. Carraro and Siniscalco (1993). In our model governments do not play Nash. Instead they do or do not take action depending on their (non)commitment in a Kyoto-type international agreement.
} 
the firm in country $F$ does not incur any extraction costs. While this assumption is not realistic ${ }^{6}$ it is not central for the qualitative conclusions to be derived.

The first-order conditions of maximizing (7), (8) and (9) read, respectively,

$$
\begin{array}{rlr}
\pi_{1}=p_{x 1} X_{e_{A 1}}^{A}-p_{e 1}>0 & \text { and } & p_{x 2} X_{e_{A 2}}^{A}=p_{e 2}, \\
p_{x 1} X_{e_{N 1}}^{N}=p_{e 1} & \text { and } & p_{x 2} X_{e_{N 2}}^{N}=p_{e 2}, \\
p_{e 1}=p_{e 2} . &
\end{array}
$$

We assume $\pi_{1}>0$ in (10) because we consider an emissions cap $\bar{e}_{A 1}$ that is strictly binding in the relevant range of equilibrium prices. ${ }^{7}$ As noted above, $\pi_{2} \equiv 0$ if (6a) applies and $\pi_{2}>0$ if the relevant constraints are given by (6b). In case of $p_{e 1} \neq p_{e 2}$ the fossil-fuel selling firm would sell all fossil fuel either in the first or in the second period generating an excess demand in that period in which its supply is zero. Hence equation (11) represents a necessary (arbitrage) condition for equilibrium. ${ }^{8}$ In equilibrium, ${ }^{9}$ the firm is indifferent between selling its fossil fuel in period 1 or 2 .

The consumer maximizes utility (2) subject to her budget constraint ${ }^{10}$

$$
\sum_{t} p_{x t} x_{i t}=\left\{\begin{array}{l}
=\Pi^{A *}+\pi_{1} \bar{e}_{A 1} \\
=\Pi^{i *}
\end{array} \quad \text { for } i=F, N,\right.
$$

which yields

$$
\frac{U_{x_{i 1}}}{U_{x_{i 2}}}=\frac{p_{x 1}}{p_{x 2}} \quad \text { for } i=A, F, N
$$

We have thus completed the description of the model and are ready for studying the impact of policy changes in country $A$. In the next Section 3 we will consider the policy scenario (6a) and investigate the allocative effects when country $A$ tightens its emissions cap $\bar{e}_{A 1}\left(\mathrm{~d} \bar{e}_{A 1}<0\right)$. Section 4 explores the effects of enlarging the group of abating countries and after that we will turn to the scenario (6b) in Section 5 and investigate the impact of the policy changes $\left(\mathrm{d} \bar{e}_{A 1}<0\right.$ and $\left.\mathrm{d} \bar{e}_{A 2}=0\right)$ as well as $\left(\mathrm{d} \bar{e}_{A 1}=0\right.$ and $\left.\mathrm{d} \bar{e}_{A 2}<0\right)$.

\footnotetext{
${ }^{6}$ In fact, zero extraction costs tend to favor carbon leakage because it makes the supply of fossil fuel perfectly elastic. For the consideration of stock-dependent extraction costs see Sinn (2008).

${ }^{7}$ Sufficient for (10) and (11) are the regularity conditions $\lim _{e_{j t} \rightarrow 0} X_{e_{j t}}^{j}=\infty$ and $\lim _{e_{j t} \rightarrow \infty} X_{e_{j t}}^{j}=0$ for $j=A, N$ and $t=1,2$ which we assume to hold.

${ }^{8}$ The Hotelling rule requires the market rate of interest to equal the rate of increase in the price of the natural resource. Since in our model the market rate of interest is zero by assumption, (12) is a simplified version of the Hotelling rule.

${ }^{9}$ We rule out the polar case of $p_{e 1}=p_{e 2}=0$ in equilibrium, a necessary condition for which is that the fossil-fuel demand is finite at $p_{e 1}=p_{e 2}=0$. This is the only case where part of the resource stock might be left in the ground.

${ }^{10} \mathrm{In}(12) \Pi^{i *}$ is the maximum profit of the firm in country $i$. The budget constraints can be rearranged to $\sum_{t}\left[p_{x t}\left(x_{i t}^{s}-x_{i t}\right)-p_{e t} e_{i t}\right]$ for $i=A, N$ and $\sum_{t}\left(p_{e t} e_{F t}-p_{x t} x_{F t}\right)=0$ which turn out to be the countries' intertemporal trade balances.
} 


\section{Tightening the emissions cap in the first period}

Consider a competitive equilibrium in the three-country model (1) - (5), (6a), (7) - (14) in which the constraint (6a) is strictly binding and suppose the emissions cap $\bar{e}_{A 1}$ is tightened: ${ }^{11}$ $\hat{\bar{e}}_{A 1}:=\frac{\mathrm{d} \bar{e}_{A 1}}{\bar{e}_{A 1}}<0$. Carbon leakage is said to occur if $\hat{e}_{N 1} / \hat{\bar{e}}_{A 1}<0$. Carbon leakage is particularly severe, if the reduction of carbon emissions in country $A$ is overcompensated by the (induced) increase in carbon emissions in country $N$, i.e. if $\hat{e}_{F 1} / \hat{\bar{e}}_{A 1}<0$. In such a situation tightening the emissions control in country $A$ increases total carbon emissions in period 1, which is called 'green (policy) paradox'. Country $A$ 's effort of fighting global warming actually turns out to promote global warming. ${ }^{12}$

We aim at investigating the conditions under which the green paradox occurs in the analytical framework developed in Section 2. For that purpose we first determine the displacement effect of $\hat{\bar{e}}_{A 1} \neq 0$ on the intertemporal market for fossil fuel: ${ }^{13,14}$

$$
\begin{aligned}
\hat{e}_{F 1} \cdot e_{F 1} & =\underbrace{\bar{e}_{A 1} \hat{\bar{e}}_{A 1}}_{[1]}-\underbrace{\frac{p_{e 1} e_{N 1}\left|\eta_{N 1}\right| \bar{e}_{A 1}}{\gamma_{p}+p_{e 1} e_{N 1}\left|\eta_{N 1}\right|}}_{[2]} \hat{\bar{e}}_{A 1}-\underbrace{\frac{\gamma_{p} e_{N 1}\left|\eta_{N 1}\right|}{\gamma_{p}+p_{e 1} e_{N 1}\left|\eta_{N 1}\right|}}_{[3]} \hat{p}_{x 2} \\
& =\frac{\gamma_{p} \bar{e}_{A 1}}{\gamma_{p}+p_{e 1} e_{N 1}\left|\eta_{N 1}\right|} \hat{\bar{e}}_{A 1}-\frac{\gamma_{p} e_{N 1}\left|\eta_{N 1}\right|}{\gamma_{p}+p_{e 1} e_{N 1}\left|\eta_{N 1}\right|} \hat{p}_{x 2} .
\end{aligned}
$$

In (15), $\eta_{N 1}:=\frac{X_{e_{N 1}}^{N}}{e_{N 1} X_{e_{N 1} e_{N 1}}^{N}}<0$ is country $N^{\prime} s$ price elasticity of demand for fossil fuel in period 1 and $\gamma_{p}:=-p_{e 1}\left(e_{A 2} \eta_{A 2}+e_{N 2} \eta_{N 2}\right)>0$. We are in the position to show

Proposition 1. If (6a) holds and the abating country A tightens its emissions cap $\left(\hat{\bar{e}}_{A 1}<0\right)$,

- the second-period price of the consumption good falls $\left(\hat{p}_{x 2}<0\right)$,

- the fossil fuel price falls $\left(\hat{p}_{e 1}=\hat{p}_{e 2}<0\right)$,

- and the first-period emissions either decline by less than $\mathrm{d} \bar{e}_{A 1}$ (which corresponds to a positive leakage rate less than 100\%; $\left.\frac{\mathrm{d} e_{F 1}}{\mathrm{~d} \bar{e}_{A 1}} \in\right] 0,1[$ ) or they increase (green paradox; $\left.\frac{\mathrm{d} e_{F 1}}{\mathrm{~d} \bar{e}_{A 1}}<0\right)$.

Proof. Contrary to the ascertion suppose that $\hat{p}_{x 2} / \hat{\bar{e}}_{A 1}<0$. In that case (15b) yields $\hat{e}_{F 1} / \hat{\bar{e}}_{A 1}>0$ and $\hat{e}_{F 2} / \hat{\bar{e}}_{A 1}<0$ (due to $e_{F 1} \hat{e}_{F 1}+e_{F 2} \hat{e}_{F 2}=0$ ). Differentiation of (1), (4),

\footnotetext{
${ }^{11}$ Throughout the paper the 'hat variables' are defined as $\hat{y}=\mathrm{d} y / y$.

${ }^{12}$ In view of (5) we have sign $\left(\hat{e}_{F 1} / \hat{\bar{e}}_{A 1}\right)=-\operatorname{sign}\left(\hat{e}_{F 2} / \hat{\bar{e}}_{A 1}\right)$. As the goal of climate policy is to delay the consumption of fossil fuel, tightening the emissions cap $\hat{\bar{e}}_{A 1}$ promotes that goal only if $\hat{e}_{F 1} / \hat{\bar{e}}_{A 1}>0$.

${ }^{13}(15 \mathrm{a})$ is derived in the Appendix B.

${ }^{14}$ Throughout the rest of the paper good $X$ in period 1 is chosen as numeraire $\left(p_{x 1} \equiv 1\right)$.
} 
$q^{s}:=\frac{x_{A 1}^{s}+x_{N 1}^{s}}{x_{A 2}^{s}+x_{N 2}^{s}}$ and then using (10) - (12) yields

$$
\begin{aligned}
\hat{x}_{A 1}^{s} x_{A 1}^{s}+\hat{x}_{N 1}^{s} x_{N 1}^{s} & =p_{e 1}\left(\hat{\bar{e}}_{A 1} \bar{e}_{A 1}+\hat{e}_{N 1} e_{N 1}\right)+\pi_{1} \hat{\bar{e}}_{A 1} \bar{e}_{A 1}, \\
\hat{x}_{A 2}^{s} x_{A 2}^{s}+\hat{x}_{N 2}^{s} x_{N 2}^{s} & =\frac{p_{e 2}}{p_{x 2}}\left(\hat{e}_{A 2} e_{A 2}+\hat{e}_{N 2} e_{N 2}\right), \\
e_{F t} \hat{e}_{F t} & =\hat{e}_{A t} e_{A t}+\hat{e}_{N t} e_{N t} \quad t=1,2, \\
\hat{q}^{s} & =\frac{\hat{x}_{A 1}^{s} x_{A 1}^{s}+\hat{x}_{N 1}^{s} x_{N 1}^{s}}{x_{A 1}^{s}+x_{N 1}^{s}}-\frac{\hat{x}_{A 2}^{s} x_{A 2}^{s}+\hat{x}_{N 2}^{s} x_{N 2}^{s}}{x_{A 2}^{s}+x_{N 2}^{s}} .
\end{aligned}
$$

Taking advantage of $\hat{e}_{F 1} / \hat{\bar{e}}_{A 1}>0, \hat{e}_{F 2} / \hat{\bar{e}}_{A 1}<0$, and (18) in (16) and (17), we get $\left(\hat{x}_{A 1}^{s} x_{A 1}^{s}+\right.$ $\left.\hat{x}_{N 1}^{s} x_{N 1}^{s}\right) / \hat{\bar{e}}_{A 1}>0$ and $\left(\hat{x}_{A 2}^{s} x_{A 2}^{s}+\hat{x}_{N 2}^{s} x_{N 2}^{s}\right) / \hat{\bar{e}}_{A 1}<0$ and hence $\hat{q}^{s} / \hat{\bar{e}}_{A 1}>0$ from (19). Consider now the demand side and observe that (14) implies

$$
\widehat{\left(\frac{x_{i 1}}{x_{i 2}}\right)}=: \hat{q}_{i}^{d}=\sigma_{i} \hat{p}_{x 2} \quad \text { for } i=A, F, N \text {. }
$$

Since all utility functions are assumed to be identical we have $\sigma_{i}=\sigma$ and $q_{i}^{d}=q^{d}$ for $i=A, F, N$. Under this condition (20) implies $\hat{q}^{d} / \hat{\bar{e}}_{A 1}=\sigma \cdot\left(\hat{p}_{x 2} / \hat{\bar{e}}_{A 1}\right)<0$ for $\hat{p}_{x 2} / \hat{\bar{e}}_{A 1}<0$. $\left(\hat{q}^{s}-\hat{q}^{d}\right) / \hat{\bar{e}}_{A 1}>0$ follows. However, $\left(\hat{q}^{s}-\hat{q}^{d}\right) / \hat{\bar{e}}_{A 1}=0$ is a necessary equilibrium condition. This contradiction proves the claim $\hat{p}_{x 2} / \hat{\bar{e}}_{A 1}>0 . \hat{p}_{e 1} / \hat{\bar{e}}_{A 1}>0$ is straightforward from $\hat{p}_{x 2} / \hat{\bar{e}}_{A 1}>0$ and (B6), and $\mathrm{d} e_{F 1} / \mathrm{d} \bar{e}_{A 1}<1$ follows from (15b).

Proposition 1 conveys the important messages that if country $A$ tightens its firstperiod emissions cap the world market price of fossil fuel (in terms of first-period consumption) falls and first-period consumption becomes more expensive relative to second-period consumption. ${ }^{15}$ For both reasons it is profitable for the firms in country $N$ to expand their output and hence their fossil fuel consumption.

Equation (15a) specifies the first-period emissions reduction induced by tightening the cap $\bar{e}_{A 1}\left(\hat{\bar{e}}_{A 1}<0\right)$. The term [1] is the (partial) direct effect which would imply zero leakage in the absence of market adjustments. The terms [2] and [3] represent leakage effects. [2] captures the increase in $e_{F 1}$ caused by the drop in $p_{e 1}$, if $p_{x 2}$ is (hypothetically) kept constant. The increase in $e_{F 1}$ due to [2] is the smaller the more price elastic the fuel demand of the countries $A$ and $N$ are in period $2\left(\gamma_{p}\right.$ larger) and the more price elastic the fuel demand of country $N$ is in period 1 ( $\left|\eta_{N 1}\right|$ larger). Observe that the effect [2] generates carbon leakage but does not lead to the green paradox since the term $\frac{\gamma_{p}}{\gamma_{p}-p_{e 1} e_{N 1} \eta_{N 1}}$ in (15b) is positive but less than one. However, the effect [3] exacerbates carbon leakage (since $\hat{p}_{x 2} / \hat{\bar{e}}_{A 1}>0$ ) and creates the possibility of the green paradox which will then occur if and only if the effect [3] is sufficiently strong. As response to tightening the cap, the increase in the first-period consumption of fossil fuel, $e_{F 1}$, due to [3] is the larger the more

\footnotetext{
${ }^{15}$ This observation is clearly equivalent to the statement that second-period consumption becomes less expensive relative to first-period consumption which we have chosen as numeraire.
} 
price elastic is the aggregate fuel demand in period $2\left(\gamma_{p}\right.$ larger $)$, the more price elastic is the fuel demand of country $N$ in period 1 ( $\left|\eta_{N 1}\right|$ larger), and the greater is the decline in the second-period price of the consumption good, $\hat{p}_{x 2} / \hat{\bar{e}}_{A 1}$. The role of effect [3] is to equilibrate the markets for the consumption good in both periods while maintaining the equilibrium in the markets for fossil fuel through an appropriate reduction in $p_{e 1}$. This observation highlights that the effect [3] emerges in our model because it contains a full set of competitive (future) markets all of which are required to clear.

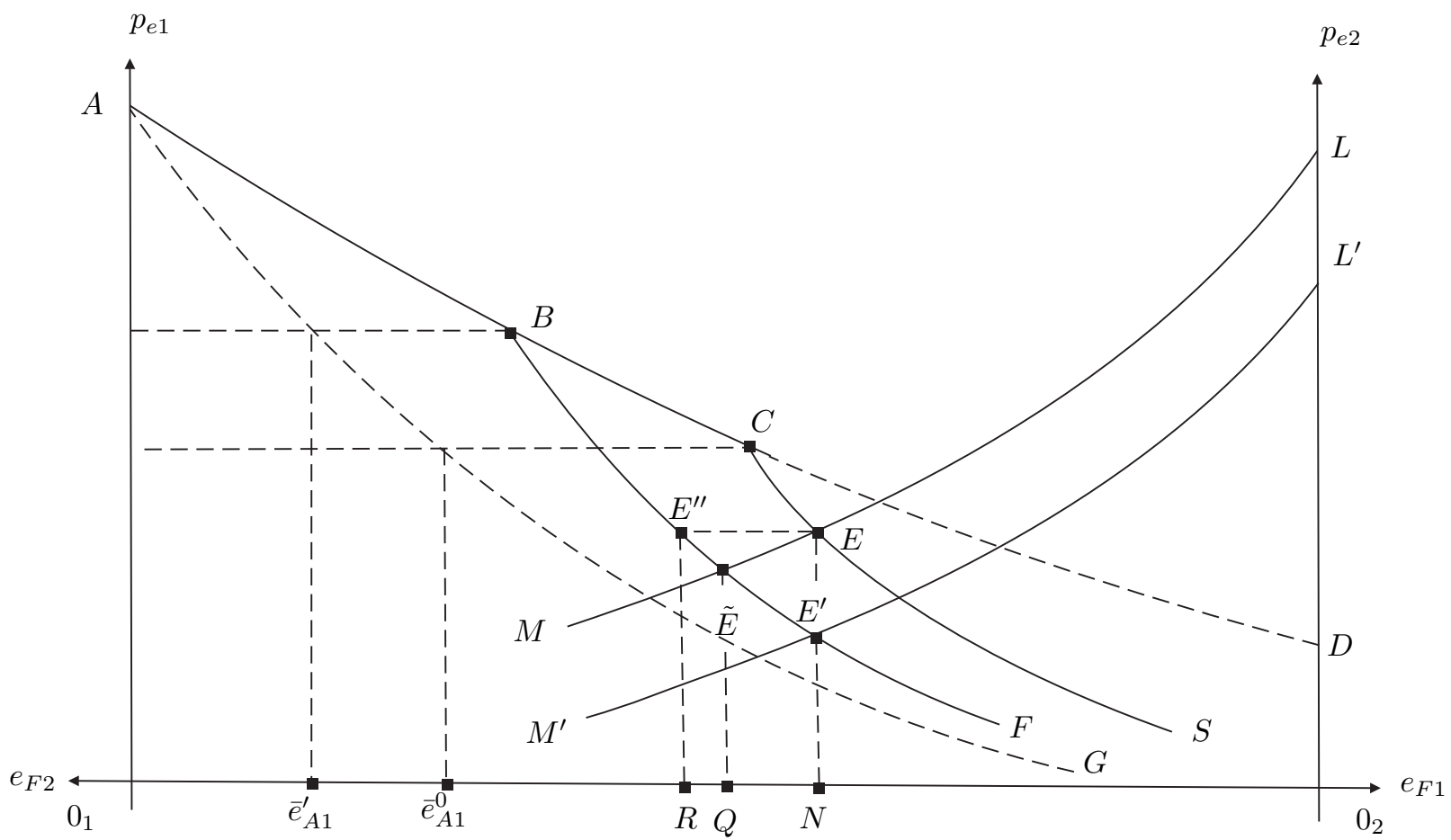

Figure 1: Impact of tightening the emissions cap in period 1

The effects of tightening the cap $\bar{e}_{A 1}$ are illustrated in Figure 1 . Let $A G$ be the first-period fuel demand curve of country $A$ when no cap is applied and let $A B C D$ be the aggregate first-period demand curve (where country $N$ 's demand is horizontally added to country $A$ 's demand). The line $A C S$ is the aggregate first-period demand for fossil fuel when country $A$ 's emissions cap is $\bar{e}_{A 1}^{0}$. With that cap in place, aggregate demand is still unconstrained in the segment $A B C$. The segment $C D$, however, is now replaced by the line $C S$. For fossil-fuel prices less than $0_{1} T$, country $A$ 's fuel demand is constant at $\bar{e}_{A 1}^{0}$, while country $N$ 's demand expands with sinking prices along $C S$.

According to (10) and (11) the aggregate second-period demand for fossil fuel can be depicted in Figure 1 only for some predetermined price $p_{x 2}$. Suppose, $p_{x 2}^{0}$ is the equilibrium value of $p_{x 2}$ when country $A$ has fixed the emissions cap $\bar{e}_{A 1}^{0}$ and let $L M$ represent the aggregate fuel demand in period 2 when $p_{x 2}=p_{x 2}^{0}$ prevails and when second-period emissions are not capped. According to (12) point $E$ in Figure 1 then characterizes the equilibria in 
the periods 1 and 2 of the world markets for fossil fuel where $p_{e 1}^{0}=p_{e 2}^{0}=N E$.

Suppose now country $A$ tightens its emissions cap from $\bar{e}_{A 1}^{0}$ to $\bar{e}_{A 1}^{\prime}, \bar{e}_{A 1}^{\prime}<\bar{e}_{A 1}^{0}$. As a consequence, the curve of the first-period aggregate fuel demand shifts from $A C S$ to $A B F$. In Figure 1, the emissions reduction $\bar{e}_{A 1}^{0}-\bar{e}_{A 1}^{\prime}$ is given by $E E^{\prime \prime}=N R$ representing the partial effect [1] in equation (15a). If the second-period demand curve $L M$ remained unchanged (which would be the case if and only if $p_{x 2}$ remained unchanged) the equilibrium shifts from $E$ to $\tilde{E}$. The resultant increase in $e_{F 1}$ by $Q R$ (from $0_{1} R$ to $0_{1} Q$ ) corresponds to the partial effect [2] in equation (15a). In point $\tilde{E}$ in Figure 1 the markets for fossil fuel are cleared in both periods but the commodity markets are still in disequilibrium. We know from Proposition 1 that the second-period price of the consumption good must decline $\left(\hat{p}_{x 2} / \hat{\bar{e}}_{A 1}>0\right)$ in order to clear the commodity markets. The drop in $p_{x 2}$ shifts downward the demand curve $L M$ to e.g. $L^{\prime} M^{\prime}$. The new equilibrium point is $E^{\prime}$ and the increase $N Q$ in first-period emissions, $e_{F 1}$, involved in the move from $\tilde{E}$ to $E^{\prime}$ corresponds to the partial effect [3] in equation $(15 \mathrm{a}){ }^{16}$

Approaching the leakage issue from the perspective of the non-abating country $N$ we observe that the increase in first-period emissions of country $N$ is analytically given by equation (A14) in the Appendix A,

$$
\underset{(+)}{\hat{e}_{N 1}}=\underset{(-)(-)}{\eta_{N 1}} \hat{p}_{e 1} .
$$

Hence the first-period emissions of country $N, \hat{e}_{N 1}$, are positive and the greater, the greater in absolute value is the price elasticity of demand for fossil fuel of the firm in country $N$ and the greater is the decline in the fossil-fuel price, $\hat{p}_{e 1}<0$. As illustrated above, we can decompose the reduction in the fossil-fuel price as follows:

$$
\hat{p}_{e 1}=\underbrace{\left.\hat{p}_{e 1}\right|_{\mathrm{d} p_{x 2}=0}}_{[1]}+\underbrace{\left.\hat{p}_{e 1}\right|_{\mathrm{d} p_{x 2}<0}}_{[2]}
$$

The components [1] and [2] in (21) are both negative. The partial effect [1] has been shown to generate leakage at a rate less than $100 \%$. The partial effect [2] aggravates total leakage and thus has the potential to raise the leakage rate above 100\% (green paradox).

According to (15b), Proposition 1 and the preceding discussion the price change $\hat{p}_{x 2}$ is the key determinant for the green paradox because - as we have illustrated with the help of Figure 1 - it is crucial how large the reduction in $p_{x 2}$ must be to bring about the necessary equilibrium condition $\hat{q}^{d}=\hat{q}^{s}$. To better understand the relation between $\hat{p}_{x 2}$ and $\hat{q}^{d}$ we

\footnotetext{
${ }^{16}$ The shift of the second-period demand curve from $L M$ to $L^{\prime} M^{\prime}$ happens to be chosen such that carbon leakage exactly offsets country $A$ 's emissions reduction $\bar{e}_{A 1}^{0}-\bar{e}_{A 1}^{\prime}$.
} 
resort to the class of CES utility functions that are homogeneous of degree $b>0$ (and hence homothetic):

$$
U\left(x_{i 1}, x_{i 2}\right)=\left(a_{1} x_{i 1}^{-e}+a_{2} x_{i 2}^{-e}\right)^{-\frac{b}{e}}
$$

where $a_{1}>0, a_{2}>0$ and $e:=(1-\sigma) / \sigma$. When combined with (14), standard calculations lead to

$$
q^{d}=\left(\frac{a_{2} p_{x 2}}{a_{1}}\right)^{\sigma}=: Q^{d}\left(p_{x 2}, \sigma\right) .
$$

The equilibrium condition is

$$
Q^{d}\left(\begin{array}{cc}
p_{x 2}, \underset{(+)}{(+)} \\
(+)
\end{array}\right)=Q^{s}\left(\begin{array}{c}
\bar{e}_{A 1}, p_{x 2} \\
(+) \\
(-)
\end{array}\right),
$$

where the function $Q^{s}$ is implicitly determined in (19). ${ }^{17}$ The equilibrium price $p_{x 2}$ is uniquely determined by (24) and it obviously depends on both $\sigma$ and $\bar{e}_{A 1}$.

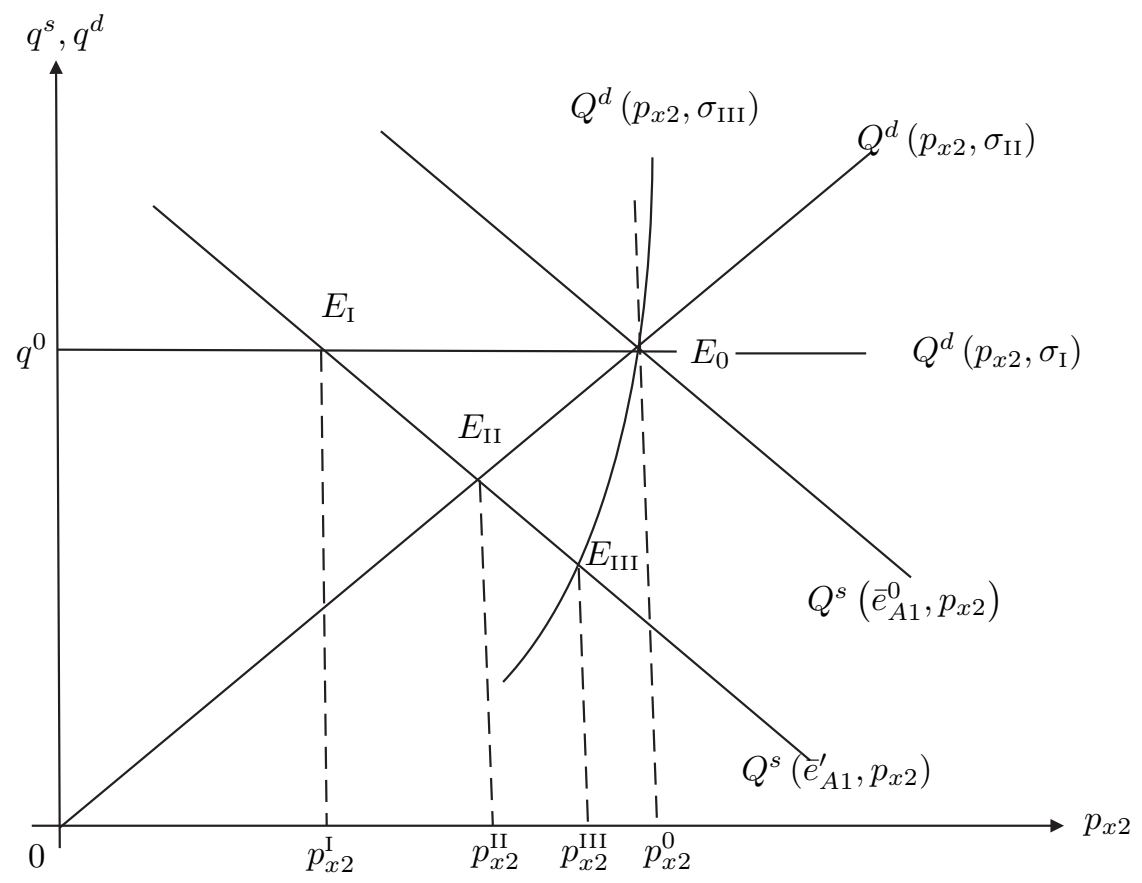

Figure 2: Changes in $p_{x 2}$ when the cap is tightened from $\bar{e}_{A 1}^{0}$ to $\bar{e}_{A 1}^{\prime}$ depending on the size of $\sigma\left(\sigma_{\mathrm{I}}=0, \sigma_{\mathrm{II}}=1, \sigma_{\mathrm{III}}\right.$ very large $)$

In Figure 2 we have plotted the graphs of $Q^{d}$ for alternative values of $\sigma: \sigma_{\mathrm{I}}=0, \sigma_{\mathrm{II}}=1$ and $\sigma_{\text {III }}$ very large. Figure 2 also contains the graphs of the function $Q^{s}$ for $\bar{e}_{A 1}=\bar{e}_{A 1}^{0}$ and for $\bar{e}_{A 1}=\bar{e}_{A 1}^{\prime}<\bar{e}_{A 1}^{0}$. Starting from an initial equilibrium ${ }^{18} E_{0}$ and tightening the emissions

\footnotetext{
${ }^{17}$ The signs of the derivatives of the function $Q^{s}$ are shown in the Appendix C.

${ }^{18}$ We have demonstrated in (24) that the equilibrium values of $p_{x 2}$ and $q$ depend on the parameters $\bar{e}_{A 1}$ and $\sigma$. The only reason for taking $E_{0}$ in Figure 2 as one and the same equilibrium point for alternative values of $\sigma$ is to ease the exposition.
} 
cap from $\bar{e}_{A 1}^{0}$ to $\bar{e}_{A 1}^{\prime}$ leads to new equilibria $E_{0}, E_{\mathrm{I}}, E_{\mathrm{II}}$ or $E_{\mathrm{III}}$. The abscissa shows that the lower the substitution elasticity the greater is the reduction in the price $p_{x 2}$.

To make further progress in exploring the conditions for the green paradox we calculate in the Appendix D the total impact of tightening the abating country's cap $\left(\hat{\bar{e}}_{A 1}<0\right)$ on first-period emissions $\left(\hat{e}_{F 1}\right)$ and show that

$$
\frac{\hat{e}_{F 1}}{\hat{\bar{e}}_{A 1}} \gtreqless 0 \quad \Longleftrightarrow \sigma \cdot\left(x_{A 1}^{s}+x_{N 1}^{s}\right) \gtreqless-\pi_{1} e_{N 1} \eta_{N 1} \text {. }
$$

Proposition 2. $\quad$ Suppose (6a) holds and the abating country A tightens its cap $\left(\hat{\bar{e}}_{A 1}<\right.$ $0)$. Then the green paradox occurs if and only if $\sigma<\bar{\sigma}:=\frac{\pi_{1} e_{N 1}\left|\eta_{N 1}\right|}{x_{A 1}^{s}+x_{N 1}^{s}}$.

By definition of $\bar{\sigma}$ in Proposition 2, the green paradox is the more likely to occur for any given value of the substitution elasticity $\sigma$ the smaller is first-period world production $x_{A 1}^{s}+x_{N 1}^{s}$, the greater in absolute value is the first-period price elasticity of demand for fossil fuel in the non-abating country, the tighter is the abating country's cap (because tightening the cap raises the permit price $\pi_{1}$ ) and the greater the first-period emissions of the non-abating country, $e_{N 1}$, are.

For Leontief utility functions $(\sigma=0)$ the green paradox occurs but it does not occur in the case of utility functions exhibiting sufficiently large substitution elasticities. If we consider the class of isoelastic utility functions

$$
U\left(x_{i 1}, x_{i 2}\right)= \begin{cases}\frac{\mu x_{i 1}^{1-\eta}}{1-\eta}+\frac{1}{1+\rho} \cdot \frac{\mu x_{i 2}^{1-\eta}}{1-\eta} & \text { for } \quad \mu>0, \eta \neq 1 \\ \ln x_{i 1}+\frac{1}{1+\rho} \ln x_{i 2} & \text { for } \quad \eta=1\end{cases}
$$

where $\rho$ is a positive pure rate of time preference, we find that $\eta=\frac{1}{\sigma}$ and hence $\sigma \rightarrow \infty$ if and only if $\eta \rightarrow 0$. No doubt, isoelastic utility functions with $\eta \rightarrow 0$ are unrealistic as well as Leontief utility functions. Yet these polar cases are of some interest because they provide sufficient conditions for the existence and non-existence, respectively, of the green paradox. ${ }^{19}$ The main message of Proposition 2 in combination with Figure 2 is that the lower the substitution elasticity the greater is the price effect $\hat{p}_{x 2} / \hat{\bar{e}}_{A 1}$ and the more likely is the green paradox.

As pointed out above the size of the threshold value $\bar{\sigma}$ defined in Proposition 2 also depends among other things on the size of the price elasticity of demand for fossil fuel, $\eta_{N 1}$, which is entirely technology-determined. To get more information on the interaction of demand and supply conditions yielding the green paradox we parametrize the production

\footnotetext{
${ }^{19}$ It is also worth mentioning that the condition for the green paradox does not hinge upon the pure rate of time preference.
} 
function by

$$
\left.X^{i}\left(e_{i t}\right)=e_{i t}^{\theta_{i t}}, \quad \theta_{i t} \in\right] 0,1[\text { for } i=A, N .
$$

For production functions (27), the equivalence (25) simplifies to

$$
\frac{\hat{e}_{F 1}}{\hat{\bar{e}}_{A 1}} \gtreqless 0 \Longleftrightarrow \sigma \cdot\left(1-\theta_{N 1}\right) \gtreqless \frac{\pi_{1} e_{N 1}}{x_{A 1}^{s}+x_{N 1}^{s}}=: \gamma_{\theta 1}
$$

From (28) we infer (see the Appendix E)

Proposition 3. Suppose (6a) holds, the production function $X^{N}\left(e_{N 1}\right)$ from (1) is specified by (27) and the abating country A tightens its cap $\left(\hat{\bar{e}}_{A 1}<0\right)$.

(i) Then the green paradox occurs, if and only if $\sigma \cdot\left(1-\theta_{N 1}\right)<\gamma_{\theta 1}$.

(ii) The green paradox does not occur, either

(a) if $p_{e 1} \geq \pi_{1}$ and $\sigma \cdot\left(1-\theta_{N 1}\right) \geq 1$ or

(b) if $p_{e 1} \geq \pi_{1}, \bar{e}_{A 1} \geq e_{N 1}$ and $\sigma \cdot\left(1-\theta_{N 1}\right) \geq \frac{1}{3}$.

Proposition 3 highlights the relevance for the green paradox of the production technology in country $N$ and of the interaction of supply and demand conditions. It conforms with our intuition that a highly elastic demand for fossil fuel in country $N$ is conducive to the green paradox. That elasticity is the higher the closer to one is the production parameter $\theta_{N 1}$, i.e. the more the production function tends to be linear. Yet even if $\theta_{N 1}$ is small the green paradox occurs according to Proposition 3(i) if $\sigma$ is sufficiently small. On the other hand, Proposition 3(ii) states conditions under which the green paradox does not occur. Proposition 3(ii) does not imply, however, that avoiding the green paradox requires $\sigma>1$, because the pertaining conditions are sufficient but not necessary. To sum up, according to Proposition 3 the green paradox depends on the order of magnitude of the parameters $\sigma$ and $\theta_{N 1}$. This calls for a thorough discussion of the empirical estimates of those parameters which is, however, beyond the scope of the present paper.

\section{Enlarging the group of abating countries}

Up to now we have not made any explicit assumption about the size of the abating country $A$ compared to the size of the non-abating country $N .{ }^{20}$ We will do so now in the simplest

\footnotetext{
${ }^{20}$ Note, however, that $\bar{\sigma}$ in Proposition 2 can be rewritten as $\bar{\sigma}:=-\pi_{1} \eta_{N 1} \varphi$, where $\varphi:=e_{N 1} /\left(x_{A 1}^{s}+x_{N 1}^{s}\right)$ is positively correlated to the non-abating country's share in world production.
} 
possible way by introducing a fixed world endowment of an immobile (internationally nontradable) factor (e.g. land), $\bar{\ell}=1$, where $\ell \in] 0,1[$ and $1-\ell$, respectively, are the inputs of land employed in the countries $A$ and $N$. To further simplify the exposition, suppose the production functions are Cobb-Douglas such that for $t=1,2$

$$
x_{A t}^{s}=e_{A t}^{\alpha} \ell^{1-\alpha} \quad \text { and } \quad x_{N t}^{s}=e_{N t}^{\alpha}(1-\ell)^{1-\alpha},
$$

where $\alpha \in] 0,1[$. Note first that in the absence of emissions capping the aggregate demand functions for fossil fuel (in either period) are independent of $\ell$. This is easily verified by combining (29) with the profit maximizing condition $X_{e_{i t}}^{i}=p_{t}$ for $t=1,2$ (with $p_{1}=p_{e 1}$ and $\left.p_{2}=p_{e 2} / p_{x 2}\right)$ to calculate the countries' fuel demand functions as

$$
e_{A t}=\ell\left(\frac{\alpha}{p_{t}}\right)^{\frac{1}{1-\alpha}} \quad \text { and } \quad e_{N t}=(1-\ell)\left(\frac{\alpha}{p_{t}}\right)^{\frac{1}{1-\alpha}} .
$$

Adding up these equations shows that for any given $p_{t}$ the sum $e_{A t}+e_{N t}$ remains unchanged when $\ell$ is varied. We interpret an increase in $\ell$ as new countries joining the group of abating countries which we continue to address as "country $A$ ", though. Differentiating country $A$ 's fuel demand function yields

$$
\hat{p}_{t}=\left(\hat{\ell}-\hat{\bar{e}}_{A t}\right)(1-\alpha) \text {. }
$$

If we would increase $\ell$ and would keep constant the emissions cap $\bar{e}_{A 1}$ we would combine enlarging the group with tightening the cap for all members of the group. To avoid such mixed strategy we will consider a scenario where the enlargement of country $A, \hat{\ell}>0$, is combined with a proportional relaxation of the cap, $\hat{\ell}=\hat{\bar{e}}_{A t}>0$. That procedure appears plausible since it implies $\hat{p}_{e 1}=0$ so that the countries entering the abatement coalition commit to the same constraint as the old members.

Figure 3 illustrates the scenario $\hat{\ell}=\hat{\bar{e}}_{A 1}>0$. The initial situation is as in Figure 1: $A G$ is country $A$ 's fuel demand curve, $A C D$ is the aggregate demand curve without cap and $A C S$ is the aggregate demand curve when the emissions cap $\bar{e}_{A 1}^{0}$ is implemented in country $A$. With the aggregate second-period demand being $L M$ the initial equilibrium is assumed to be attained in point $E$. Suppose now country $A$ 's demand curve shifts from $A G$ to $A T(\hat{\ell}>0)$ leaving the aggregate demand curve $A C D$ unchanged. $\hat{\ell}=\hat{\bar{e}}_{A 1}$ is illustrated in Figure 3 by moving from $\bar{e}_{A 1}^{0}$ to $\bar{e}_{A 1}^{1}$. The aggregate first-period demand curve associated to $\bar{e}_{A 1}^{1}$ is now given by $A C F$. Since the demand curve $A T$ is flatter than $A G$, the segment $\bar{e}_{A 1}^{0} G$ is smaller than $\bar{e}_{A 1}^{1} T$ implying that the line $C F$ is steeper than the line $C S$.

The conclusions are qualitatively similar to those we elaborated in the context of Figure 1 and are briefly described as follows: If the second-period aggregate demand curve would remain unchanged (which will not be the case) one would have carbon leakage but 


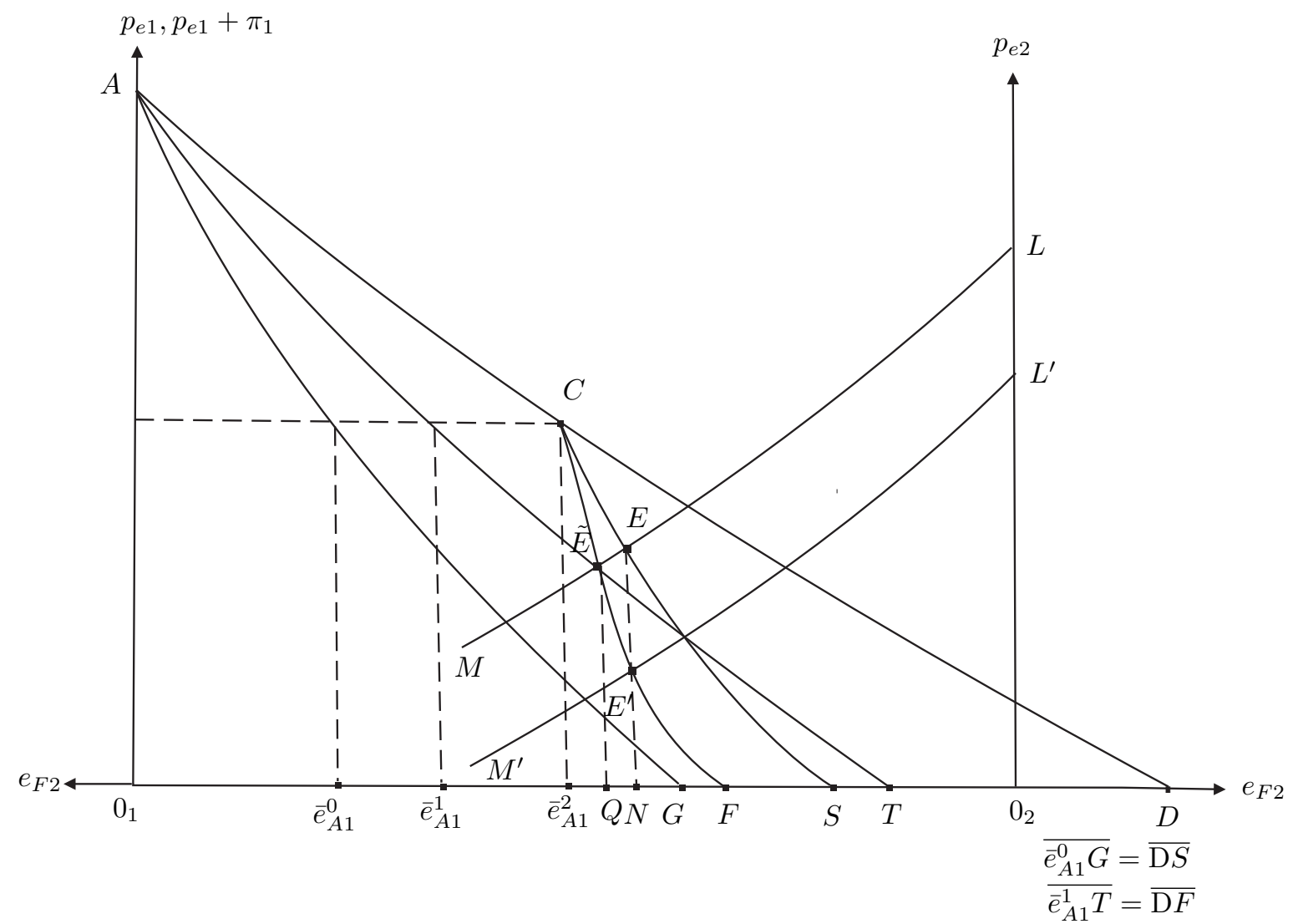

Figure 3: Enlarging the coalition of abating countries

no green paradox. Yet $p_{x 2}$ must shrink causing the $L M$ curve to shift downward to a curve such as $L^{\prime} M^{\prime}$. How far the curve $L M$ shifts down depends on the determinants elaborated in the previous Section 3. A green paradox occurs when the demand conditions require a drop in the price $p_{x 2}$ which is so strong that the second-period demand curve $L M$ is forced to shift below the line $L^{\prime} M^{\prime}$. Intuitively speaking, this is the less likely, however, the steeper is the line segment $C F$. This line segment is the steeper, in turn, the closer is $\ell$ to $\ell=1$. To see this, suppose we start from the equilibrium $E^{\prime}$ in Figure 3 (with $\left.\bar{e}_{A 1}=\bar{e}_{A 1}^{1}\right)$ and successively raise $\ell$ until $\ell=1$. Since we continue to require $\hat{\ell}=\hat{\bar{e}}_{A 1}, \ell \rightarrow 1$ obviously implies $\bar{e}_{A 1}^{1} \rightarrow \bar{e}_{A 1}^{2}$ in Figure 3 . When $\ell=1$ is reached, the aggregate first-period fuel demand curve is $A C \bar{e}_{A 1}^{2}$. In that case no leakage occurs anymore no matter how strong the downward shift of the curve $L M$ may be. We summarize these observations in

Proposition 4. Suppose the abating country A gets larger at the expense of the nonabating country $N$ and allow for relaxing the (first-period) emissions cap of country $A$ such that for the enlarged country $A$ the emissions cap is as stringent as before enlargement. Then successive enlargements tend to reduce carbon emissions and hence reduce the likelihood of a green paradoxon as well. When country A's share of world production tends toward one (making country $N$ 's size shrink to zero) the leakage rate tends toward zero. 
$\ell=1$ means that there is a global coalition in which all countries commit to reduce emissions. In that case our model turns out to be a very simple version of Sinn's (2008) model who considers a single 'aggregate' fuel-demanding country representing the global coalition of all fuel-demanding countries. In that case carbon leakage is trivially absent. However, as we know from the Kyoto protocol and the current post-Kyoto negotiations the global coalition is an unlikely scenario.

\section{Tightening the emissions cap in the second period}

In this section we assume that country $A$ regulates emissions not only in the first period, but also in the second such that the model now consists of the equations (1) - (5), (6b), (7) - (14). With two caps being implemented in country $A$ we are interested in exploring the change in overall first-period emissions either when the first-period cap is tightened or when the second-period cap is tightened. Tightening the first-period cap when caps are in operation in both periods, may differ in impact from the scenario of Section 3 where no cap was employed in the second period. Note also that tightening the second period cap is meant to model the abating country's (credible) commitment to step up its emissions reducing efforts in the "not so near" future. When exploring that scenario, carbon leakage is about how tightening the second-period cap affects total first-period emissions. Correspondingly, we will then primarily be interested in the size and sign of $\hat{e}_{F 1} / \hat{\bar{e}}_{A 2}$, while carbon leakage is defined as in Section 3 when country $A$ is subject to two caps and the first-period cap is tightened. Let us first consider the impact of $\hat{\bar{e}}_{A 1}<0$ and $\hat{\bar{e}}_{A 2}=0$.

\section{Proposition 5 .}

Suppose (6b) holds, the abating country A tightens its first-period cap $\left(\hat{\bar{e}}_{A 1}<0\right)$ and leaves its second-period cap unchanged. In qualitative terms, the conditions for carbon leakage and the green paradox are the same as under the same policy, when $e_{A 2}$ is unconstrained (as in Section 3).

As shown in the Appendix $G$, the only change necessary is replacing $\gamma_{p}:=-p_{e 1}\left(e_{A 2} \eta_{A 2}+\right.$ $\left.e_{N 2} \eta_{N 1}\right)>0$ by $\tilde{\gamma}_{p}:=-p_{e 2} e_{N 2} \eta_{N 2}>0$. With this slight modification the equation (15b) continues to hold, the equivalences in (25) and (28) remain true and hence the Propositions 1 through 3 apply.

Next, we explore the policy of tightening the second-period cap $\left(\hat{\bar{e}}_{A 2}<0\right.$ and $\left.\hat{\bar{e}}_{A 1}=0\right)$. In the Appendix $\mathrm{H}$ it is proven 
Proposition 6. If (6b) holds, if the abating country A tightens its second-period cap $\left(\hat{\bar{e}}_{A 2}<0\right)$ and leaves its first-period cap unchanged

- the second-period price of the consumption good falls $\left(\hat{p}_{x 2}<0\right)$,

- the fossil-fuel price falls $\left(\hat{p}_{e 1}=\hat{p}_{e 2}<0\right)$,

- and the first-period emissions either decline by less than $\mathrm{d} \bar{e}_{A 2}$ (which corresponds to a positive leakage rate less than 100\%; $\left.\frac{\mathrm{d} e_{F 1}}{\mathrm{~d}_{\overline{A 2}}} \in\right] 0,1[$ ) or they increase (green paradox; $\left.\frac{\mathrm{d} e_{F 1}}{\mathrm{~d} \bar{e}_{A 2}}<0\right)$.

It is remarkable that the impact of tightening the second-period cap on the second-period price of the consumption good, $p_{x 2}$, as well as on the fuel price, $p_{e 1}=p_{e 2}$, is qualitatively the same as in case of tightening the first-period cap (Propositions 1 and 5). This is also true for carbon leakage although we still have to investigate the conditions determining the extent of leakage.

Analogous to our procedure of preparing for Proposition 2 in Section 3 we calculate in the Appendix F the total impact of tightening the abating country's second-period cap $\left(\hat{\bar{e}}_{A 2}<0\right)$ on first-period emissions $\left(\hat{e}_{F 1}\right)$ and show that

$$
\frac{\hat{e}_{F 1}}{\hat{\bar{e}}_{A 2}} \gtreqless 0 \quad \Longleftrightarrow \sigma p_{x 2} \cdot\left(x_{A 2}^{s}+x_{N 2}^{s}\right) \lesseqgtr-\pi_{2} e_{N 2} \eta_{N 2} .
$$

Comparing (30) and (25) reveals a striking similarity. Yet the right side of both equivalences differs in two decisive respects: the index 1 in (25) is replaced by the index 2 in (30) and the set of inequalities in (25) is reversed ${ }^{21}$ in (30).

Closer inspection of (30) leads to

Proposition 7. Suppose (6b) holds and the abating country A tightens its secondperiod cap $\left(\hat{\bar{e}}_{A 2}<0\right)$. Then the green paradox does not occur if and only if $\sigma<\tilde{\sigma}:=$ $\frac{\pi_{2} e_{N 2}\left|\eta_{N 2}\right|}{p_{x 2}\left(x_{A 2}^{s}+x_{N 2}^{s}\right)}$.

The impact on leakage of $\sigma$ that we have established in Proposition 2 is reversed in Proposition 7. More specifically, if we tighten the first-period cap $\left(\hat{\bar{e}}_{A 1}<0\right)$ and either have the second-period cap constant $\left(\hat{\bar{e}}_{A 2}=0\right)$ or do not implement a second-period cap ( $e_{A 2}$ free) we can exclude the green paradox for sufficiently large values of $\sigma$. In contrast, under the policy of Proposition 7 the green paradox is excluded for sufficiently small values of $\sigma$.

\footnotetext{
${ }^{21}$ This reversal is exclusively due to the fact that we focus on the impact of $\hat{\bar{e}}_{A 2}$ on $e_{F 1}$ rather than on $e_{F 2}$ (because we are interested in total emissions in period 1). If we had explored the effect of $\hat{\bar{e}}_{A 2}$ on $e_{F 2}$ we could have simply referred to Section 3 interchanging the indexes 1 and 2 everywhere. This observation can be easily verified by carrying out such a swap of indexes in Figure 1.
} 
Following the procedure in Section 3 we now invoke the parametric function (27) again to complement our findings of Proposition 7. With the production function (27) the equivalence (30) turns into

$$
\frac{\hat{e}_{F 1}}{\hat{\bar{e}}_{A 2}} \gtreqless 0 \Longleftrightarrow \sigma \cdot\left(1-\theta_{N 2}\right) \lesseqgtr \frac{\pi_{e} e_{N 2}}{p_{x 2}\left(x_{A 2}^{s}+x_{N 2}^{s}\right)}=: \gamma_{\theta 2} .
$$

The information contained in (31) is summarized in

Proposition 8. Suppose (6b) holds, the production function $X^{N}\left(e_{N 2}\right)$ from (1) is specified by (27) and the abating country $A$ tightens its second-period cap $\left(\hat{\bar{e}}_{A 2}<0\right)$.

(i) Then the green paradox occurs if and only if $\sigma \cdot\left(1-\theta_{N 2}\right)<\gamma_{\theta 2}$.

(ii) The green paradox does not occur, either

(a) if $p_{e 2} \geq \pi_{2}$ and $\sigma \cdot\left(1-\theta_{N 2}\right) \geq 1$ or

(b) if $p_{e 2} \geq \pi_{2}, \bar{e}_{A 2} \geq e_{N 2}$ and $\sigma \cdot\left(1-\theta_{N 2}\right) \geq \frac{1}{3}$.

Given Proposition 7 it is not surprising anymore that the impact of $\sigma$ and $\theta$ on carbon leakage in the scenario of Proposition 3 is reversed in Proposition 8. All parameter changes that make the green paradox more likely when the cap is tightened in period 1 work in opposite direction when the cap is tightened in period 2. In particular, the green paradox will likely occur when the emissions control is strengthened in the second period, if it does not result from tightening the emissions cap in the first period and vice versa. ${ }^{22}$

However, in our model tightening the second-period cap does not necessarily result in the green paradox and tightening the first-period cap does not necessarily result in avoiding the green paradox. From the Propositions 2 and 7 follows that the green paradox occurs upon tightening the first-period cap as well as upon tightening the second-period cap, if and only if

$$
\frac{\pi_{2} e_{N 2}\left|\eta_{N 2}\right|}{p_{x 2}\left(x_{A 2}^{s}+x_{N 2}^{s}\right)}<\sigma<\frac{\pi_{1} e_{N 1}\left|\eta_{N 1}\right|}{x_{A 1}^{s}+x_{N 1}^{s}}
$$

Conversely, neither policy leads to the green paradox, if and only if

$$
\frac{\pi_{1} e_{N 1}\left|\eta_{N 1}\right|}{x_{A 1}^{s}+x_{N 1}^{s}}<\sigma<\frac{\pi_{2} e_{N 2}\left|\eta_{N 2}\right|}{p_{x 2}\left(x_{A 2}^{s}+x_{N 2}^{s}\right)} .
$$

\footnotetext{
${ }^{22}$ This result has an important implication for policies of tightening the emissions caps in both periods simultaneously, because the net impact on carbon leakage of simultaneous reductions in the caps of both periods is the result of "forces" working in opposite direction. More precisely, it can be shown that if $\hat{\bar{e}}_{A 1}$ and $\hat{\bar{e}}_{A 2}$ are tightened proportionally $\left(\right.$ i.e. $\hat{\bar{e}}_{A 1}=\beta \frac{\bar{e}_{A 2}}{\bar{e}_{A 1}} \hat{\bar{e}}_{A 2}$ where $\left.\beta:=\frac{e_{N 2} \lambda\left[\sigma \cdot\left(x_{A 1}^{s}+x_{N 1}^{s}\right)+\pi_{1} e_{N 1} \eta_{N 1}\right]}{e_{N 1}(1-\lambda)\left[\sigma p_{x 2} \cdot\left(x_{A 2}^{s}+x_{N 2}^{s}\right)+\pi_{2} e_{N 2} \eta_{N 2}\right]}\right)$ then there is no impact on total emissions in period 1 at all (i.e. $\frac{\hat{e}_{F 1}}{\hat{\bar{e}}_{A 2}}=0$ if $\left.\hat{\bar{e}}_{A 1}=\beta \frac{\bar{e}_{A 2}}{\bar{e}_{A 1}} \hat{\bar{e}}_{A 2}\right)$.
} 


\section{Concluding remarks}

Following Ockham's razor, we have abstracted from many real-world complexities such as extending the time horizon beyond two periods, including stock-dependent extraction costs, capital accumulation and insecure property rights. Without doubt, all of these aspects are empirically relevant but they do not appear to be at the core of the green-paradox phenomenon. To remain focused we also refrained from getting involved in the controversial though important debate on normative 'social' discounting, time preference and intertemporal inequality aversion. Our use of the demand parameters is meant to be descriptive which does not exclude extending the analysis to normative issues, of course.

We have applied the economics of intertemporal allocation of non-renewable natural resources in its simplest form and have been able to show how this approach drives the results. As is well known, in a perfectly competitive world with a full set of future markets a necessary equilibrium condition is that resource extracting and supplying firms are indifferent between selling the resource today or at any other period in the future (Hotelling rule in the wide sense; here: $p_{e 1}=p_{e 2}$ ). The requirement of clearing the market for the consumption good in both periods combined with the Hotelling rule tends to exacerbate carbon leakage when the first-period emissions cap is tightened. An interesting result is also that the impact of stepping up emissions control depends crucially on whether that policy is carried out in the first or in the second period. All determinants of carbon leakage resulting from tightening the first-period cap work in opposite direction when the second-period cap is tightened. However the extent of carbon leakage is determined by the interaction of various parameters and elasticities with the elasticity of intertemporal substitution in consumption playing a prominent role. Our model gives no unambiguous support to the proposition that tightening the second-period cap necessarily leads to the green paradox and we cannot confirm either that the green paradox results from tightening the second-period cap, if and only if it does not occur when the first-period cap is tightened.

Our analysis suggests that apart from specific characteristics of consumer preferences and production technologies it is the general equilibrium approach in a model with a complete set of perfectly competitive markets and the corresponding account of interdependence effects of markets across countries (space) and time which determines the allocation of resources including the extent of carbon leakage. Such an approach is certainly satisfactory from an intellectual viewpoint because of its consistency. However, one also needs to know how empirically relevant it is to model economic agents and policy makers who anticipate in their plans - and who trade on - perfect markets from the presence into the far future. Addressing that issue is beyond the scope of the present paper. But as fighting global change 
is an urgent empirical policy issue, assessing the reliability of theoretical guidance ought to be high on the agenda of future research, in particular, because many contributions to this issue do not integrate the economics of global change into the established intertemporal theory of nonrenewable resources.

\section{References}

Babiker, M. H. (2005), 'Climate change policy, market structure, and carbon leakage', Journal of International Economics 65, 421-445.

Burniaux, J.-M. and J. O. Martins (2000), 'Carbon emission leakage: A general equilibrium view', OECD-Economics Department Working Paper 242, OECD Publishing.

Bohm, P. (1993), 'Incomplete international cooperation to reduce $\mathrm{CO}_{2}$ emissions: alternative policies', Journal of Environmental Economics and Management 24, 258-271.

Carraro, C. and D. Siniscalco (1993), 'Strategies for the international protection of the environment', Journal of Public Economics 52, 309-328.

Copeland, B. and M.S. Taylor (2005), 'Free trade and global warming: a trade theory view of the Kyoto Protocol', Journal of Environmental Economics and Management 49, 205-234.

Farzin, Y. H. (1996), 'Optimal pricing of environmental and natural resource use with stock externalities', Journal of Public Economics 62, 31-57.

Felder, S. and T. F. Rutherford (1993), 'Unilateral $\mathrm{CO}_{2}$ reductions and carbon leakage: The consequences of international trade in oil and basic materials', Journal of Environmental Economics and Management 25, 162-176.

Gerlagh, R. O. and O. Kuik (2007), 'Carbon leakage with international technology spillovers', Nota di Lavoaro 33.2007, Fondazione Eni Enrico Mattei, Milano.

Hoel, M. (1996), 'Should a carbon tax be differentiated across sectors?', Journal of Public Economics 59, 17-32.

Hoel, M. and S. Kverndokk (1996), 'Depletion of fossil fuels and the impacts of global warming', Resource and Energy Economics 18, 115-136.

IPCC (2007), Climate Change 2007, Vol. I, II, III, Cambridge: Cambridge University Press, 2007; Synthesis Report, Vol. IV, Geneva. 
Ishikawa, J. and K. Kiyono (2006), 'Greenhouse-gas emissions controls in an open economy', International Economic Review 47, 431-450.

Ishikawa, J. and T. Okubo (2008), 'Greenhouse-gas emission controls and international carbon leakage through trade liberalization', Global COE Hi-Stat Discussion Paper Series No. 013, Hitosubashi University.

Marschinski, R., Jakob M. and O. Edenhofer (2008), 'Analysis of carbon leakage in an extended Ricardo-Viner model', Potsdam Institute for Climate Impact Research, Ms.

Paltsev, S. V. (2001), 'The Kyoto Protocol: Regional and sectoral contributions to the carbon leakage', The Energy Journal 22, 53-79.

Rubio, S. and L. Escriche (2001), 'Strategic Pigouvian taxation, stock externalities and polluting nonrenewable resources', Journal of Public Economics 79, 297-313.

Sinclair, P. J. N. (1994), 'On the trend of fossil fuel taxation', Oxford Economic Papers 46, 869-877.

Sinn, H.-W. (2008), 'Public policies against global warming', International Tax and Public Finance 15, 360-394.

Sinn, H.-W. (1982), 'Absatzsteuern, Ölförderung und das Allmendeproblem', in: H. Siebert (ed.), Reaktionen auf Energiepreissteigerungen, Frankfurt: Lang, 83-103.

Stern, N. (2006), 'The Economics of Climate Change: The Stern Review', online at http://www.hm-treasury.gov.uk.

Ulph, A. and D. Ulph (1994), 'The optimal time path of a carbon tax', Oxford Economic Papers 46, 857-868. 


\section{Appendix}

\section{A. Emissions cap in the first period: The equilibrium and the comparative statics}

The competitive equilibrium of the model is characterized by the following equations

$$
\begin{aligned}
& X_{e_{A 1}}^{A}-p_{e 1}-\pi_{1}=0 \\
& X_{e_{N 1}}^{N}-p_{e 1}=0, \\
& p_{x 2} X_{e_{i 2}}^{i}-p_{e 2}=0, \quad i=A, N \text {, } \\
& p_{e 1}-p_{e 2}=0, \\
& e_{A 1}-\bar{e}_{A 1}=0 \text {, } \\
& e_{F t}-e_{A t}-e_{N t}=0, \quad t=1,2 \text {, } \\
& \bar{e}-e_{F 1}-e_{F 2}=0 \text {, } \\
& X^{A}\left(e_{A 1}\right)-x_{A 1}-p_{e 1} e_{A 1}+p_{x 2}\left[X^{A}\left(e_{A 2}\right)-x_{A 2}\right]-p_{e 2} e_{A 2}=0, \\
& X^{N}\left(e_{N 1}\right)-x_{N 1}-p_{e 1} e_{N 1}+p_{x 2}\left[X^{N}\left(e_{N 2}\right)-x_{N 2}\right]-p_{e 2} e_{N 2}=0 \text {, } \\
& p_{e 1} e_{F 1}-x_{F 1}+p_{e 2} e_{F 2}-p_{x 2} x_{F 2}=0, \\
& X^{A}\left(e_{A 2}\right)+X^{N}\left(e_{N 2}\right)-x_{A 2}-x_{N 2}-x_{F 2}=0, \\
& \frac{U_{x_{i 2}}}{U_{x_{i 1}}}-p_{x 2}=0, \quad i=A, F, N \text {. }
\end{aligned}
$$

Without loss of generality good $X$ in period $t=1$ is chosen as numeraire $\left(p_{x 1} \equiv 1\right)$. The variables determined by (A1)-(A12) are $e_{i 1}, e_{i 2}, x_{i 1}, x_{i 2}$ for $i=A, F, N, p_{e 1}, p_{e 2}, p_{x 2}$ and $\pi_{1}$. The emissions cap $\bar{e}_{A 1}$ is treated here as an exogenous parameter. Total differentiation of (A1) - (A12) yields, after some rearrangement of terms,

$$
\begin{aligned}
e_{A 1} X_{e_{A 1} e_{A 1}}^{A} \hat{e}_{A 1}-p_{e 1} \hat{p}_{e 1}-\pi_{1} \hat{\pi}_{1} & =0, \\
\frac{\hat{e}_{N 1}}{\hat{p}_{e 1}}-\eta_{N 1} & =0, \\
\frac{\hat{e}_{i 2}}{\hat{p}_{e 2}-\hat{p}_{x 2}}-\eta_{i 2} & =0, \quad i=A, N \\
\hat{p}_{e 1}-\hat{p}_{e 2} & =0, \\
\hat{e}_{A 1}-\hat{\bar{e}}_{A 1} & =0, \\
e_{F t} \hat{e}_{F t}-e_{A t} \hat{e}_{A t}-e_{N t} \hat{e}_{N t} & =0, \quad t=1,2, \\
e_{F 1} \hat{e}_{F 1}+e_{F 2} \hat{e}_{F 2} & =0, \\
\left(X_{e_{A 1}}^{A}-p_{e 1}\right) e_{A 1} \hat{e}_{A 1}-x_{A 1} \hat{x}_{A 1}-p_{x 2} x_{A 2} \hat{x}_{A 2} & \\
-\left(e_{A 1}+e_{A 2}\right) p_{e 1} \hat{p}_{e 1}+\left[X^{A}\left(e_{A 2}\right)-x_{A 2}\right] p_{x 2} \hat{p}_{x 2} & =0,
\end{aligned}
$$




$$
\begin{aligned}
-x_{N 1} \hat{x}_{N 1}-p_{x 2} x_{N 2} \hat{x}_{N 2}-\left(e_{N 1}+e_{N 2}\right) p_{e 1} \hat{p}_{e 1} & \\
+\left[X^{N}\left(e_{N 2}\right)-x_{N 2}\right] p_{x 2} \hat{p}_{x 2} & =0 \\
\bar{e} p_{e 1} \hat{p}_{e 1}-x_{F 1} \hat{x}_{F 1}-p_{x 2} x_{F 2}\left(\hat{x}_{F 2}+\hat{p}_{x 2}\right) & =0 \\
X_{e_{A 2}}^{A} e_{A 2} \hat{e}_{A 2}+X_{e_{N 2}}^{N} e_{N 2} \hat{e}_{N 2}-x_{A 2} \hat{x}_{A 2}-x_{N 2} \hat{x}_{N 2}-x_{F 2} \hat{x}_{F 2} & =0 \\
\hat{x}_{i 2}-\hat{x}_{i 1}+\hat{p}_{x 2} \sigma_{i} & =0, \quad i=A, F, N, \text {, }
\end{aligned}
$$

where $\eta_{N 1}:=\frac{X_{e_{N 1}}^{N}}{e_{N 1} X_{e_{N 1} e_{N 1}}^{N}}<0, \eta_{i 2}:=\frac{X_{e_{i 2}}^{i}}{e_{i 2} X_{e_{i 2} e_{i 2}}^{i}}<0, \sigma_{i}:=\frac{\mathrm{d}\left(\frac{x_{i 2}}{x_{i 1}}\right)}{\frac{x_{2}}{x_{i 1}}} \cdot \frac{\frac{U x_{i 1}}{U x_{i 2}}}{\mathrm{~d}\left(\frac{U x_{i 1}}{U x_{i 2}}\right)} \geq 0$ for $i=A, F, N$.

\section{B. Derivation of (15a)}

Making use of (A24) in (A20), (A21) and (A22), respectively, yields

$$
\begin{aligned}
& \hat{x}_{A 2}=\frac{p_{x 2} \Delta x_{A 2}-x_{A 1} \sigma_{A}}{y_{A}} \hat{p}_{x 2}-\frac{p_{e 1} e_{A}}{y_{A}} \hat{p}_{e 1}+\frac{\left(X_{e_{A 1}}^{A}-p_{e 1}\right) e_{A 1}}{y_{A}} \hat{e}_{A 1}, \\
& \hat{x}_{N 2}=\frac{p_{x 2} \Delta x_{N 2}-x_{N 1} \sigma_{N}}{y_{N}} \hat{p}_{x 2}-\frac{p_{e 1} e_{N}}{y_{N}} \hat{p}_{e 1}, \\
& \hat{x}_{F 2}=\hat{p}_{e 1}-\frac{p_{x 2} x_{F 2}+x_{F 1} \sigma_{F}}{y_{F}} \hat{p}_{x 2},
\end{aligned}
$$

where $\Delta x_{i 2}:=\left(x_{i 2}^{s}-x_{i 2}\right)$ for $i=A, F, N, x_{F 2}^{s} \equiv 0, y_{i}=x_{i 1}+p_{x 2} x_{i 2}$ for $i=A, F, N$, $e_{i}=e_{i 1}+e_{i 2}$ for $i=A, N$.

Making use of (A15) and (A16) in (A23), one gets

$$
\gamma_{p}\left(\hat{p}_{x 2}-\hat{p}_{e 1}\right)=p_{x 2} x_{A 2} \hat{x}_{A 2}+p_{x 2} x_{N 2} \hat{x}_{N 2}+p_{x 2} x_{F 2} \hat{x}_{F 2},
$$

where $\gamma_{p}:=-p_{e 1}\left(e_{A 2} \eta_{A 2}+e_{N 2} \eta_{N 2}\right)>0$.

Inserting (B1) - (B3), (A1) in (B4) and rearranging terms yields

$$
\gamma_{x} \hat{p}_{x 2}-\gamma_{e} \hat{p}_{e 1}=\lambda_{A} e_{A 1} \pi_{1} \hat{\bar{e}}_{A 1}
$$

where

$$
\begin{aligned}
\gamma_{x} & :=\gamma_{p}+\lambda_{F}\left(p_{x 2} x_{F 2}+x_{F 1} \sigma_{F}\right)-\sum_{j=A, N} \lambda_{j}\left(p_{x 2} \Delta x_{j 2}-x_{j 1} \sigma_{j}\right) \\
\gamma_{e} & :=\gamma_{p}+p_{x 2} x_{F 2}-\sum_{j=A, N} \lambda_{j} p_{e 1} e_{j}
\end{aligned}
$$

where $\lambda_{i}:=\frac{p_{x 2} x_{i 2}}{x_{i 1}+p_{x 2} x_{i 2}}$ for $i=A, F, N$. Solving (A18) with respect to $\hat{e}_{F t}$, inserting this term in (A19) and making use of (A14) - (A17) we obtain

$$
-\left(\gamma_{p}-p_{e 1} e_{N 1} \eta_{N 1}\right) \hat{p}_{e 1}+\gamma_{p} \hat{p}_{x 2}=-p_{e 1} \bar{e}_{A 1} \hat{\bar{e}}_{A 1} .
$$

Next, we insert (A14) and (A17) in (A18) for $t=1$ to get

$$
\hat{e}_{F 1} e_{F 1}=\bar{e}_{A 1} \hat{\bar{e}}_{A 1}+e_{N 1} \eta_{N 1} \hat{p}_{e 1} .
$$


Finally, we solve (B6) for $\hat{p}_{e 1}$ and insert this term in (B7) to obtain (15a) after some rearrangement of terms.

\section{The function $Q^{s}\left(\bar{e}_{A 1}, p_{x 2}\right)$ and its derivatives}

We start at equation (B6) which can be rearranged to

$$
\hat{p}_{e 1}=\frac{p_{e 1} \bar{e}_{A 1} \hat{\bar{e}}_{A 1}+\gamma_{p} \hat{p}_{x 2}}{\gamma_{p}-p_{e 1} e_{N 1} \eta_{N 1}}
$$

and

$$
\hat{p}_{e 1}-\hat{p}_{x 2}=\hat{p}_{e 2}-\hat{p}_{x 2}=\frac{p_{e 1} \bar{e}_{A 1} \hat{\bar{e}}_{A 1}+p_{e 1} e_{N 1} \eta_{N 1} \hat{p}_{x 2}}{\gamma_{p}-p_{e 1} e_{N 1} \eta_{N 1}} .
$$

Differentiation of (1) yields

$$
\hat{x}_{i t}^{s}=\frac{X_{e_{i t}^{i}}^{i}}{x_{i t}^{s}} e_{i t} \hat{e}_{i t} \quad i=A, N, \quad t=1,2 .
$$

Making use of (A14) - (A17), (C1), (C2) in (C3) we get

$$
\begin{aligned}
& \hat{x}_{A 1}^{s}=X_{e_{A 1}}^{A} \bar{e}_{A 1} \hat{\bar{e}}_{A 1}, \\
& \hat{x}_{N 1}^{s}=X_{e_{N 1}}^{N} \bar{e}_{N 1} \eta_{N 1} \cdot \frac{p_{e 1} \bar{e}_{A 1} \hat{\bar{e}}_{A 1}+\gamma_{p} \hat{p}_{x 2}}{\gamma_{p}-p_{e 1} e_{N 1} \eta_{N 1}}, \\
& \hat{x}_{A 2}^{s}=X_{e_{A 2}}^{A} e_{A 2} \eta_{A 2} \cdot \frac{p_{e 1} \bar{e}_{A 1} \hat{\bar{e}}_{A 1}+p_{e 1} e_{N 1} \eta_{N 1} \hat{p}_{x 2}}{\gamma_{p}-p_{e 1} e_{N 1} \eta_{N 1}}, \\
& \hat{x}_{N 2}^{s}=X_{e_{N 2}}^{N} e_{N 2} \eta_{N 2} \cdot \frac{p_{e 1} \bar{e}_{A 1} \hat{\bar{e}}_{A 1}+p_{e 1} e_{N 1} \eta_{N 1} \hat{p}_{x 2}}{\gamma_{p}-p_{e 1} e_{N 1} \eta_{N 1}} .
\end{aligned}
$$

From (C4) - (C7) we readily infer

$$
\frac{\hat{x}_{A 1}^{s}}{\hat{p}_{x 2}}<0, \quad \frac{\hat{x}_{N 1}^{s}}{\hat{p}_{x 2}}<0, \quad \frac{\hat{x}_{A 2}^{s}}{\hat{p}_{x 2}}>0, \quad \frac{\hat{x}_{N 2}^{s}}{\hat{p}_{x 2}}>0
$$

and hence in view of (19) we get $\frac{\hat{q}_{s}}{\hat{p}_{x 2}}<0$ and $Q_{p_{x 2}}^{s}<0$, respectively. To verify $Q_{\bar{e}_{A 1}}^{s}>0$ we totally differentiate the function $Q^{s}$ to obtain

$$
\mathrm{d} q^{s}=Q_{p_{x 2}}^{s} \mathrm{~d} p_{x 2}+Q_{\bar{e}_{A 1}}^{s} \mathrm{~d} \bar{e}_{A 1}
$$

From Proposition 1 and its proof we know that $\frac{\mathrm{d} p_{x 2}}{\mathrm{~d} \bar{e}_{A 1}}>0$ and $\frac{\mathrm{d} q^{s}}{\mathrm{~d} \bar{e}_{A 1}}=\frac{\mathrm{d} q^{d}}{\mathrm{~d} \bar{e}_{A 1}}>0$. In view of (C9), $\frac{\mathrm{d} q^{s}}{\mathrm{~d} \bar{e}_{A 1}}>0$ can only be satisfied for $\frac{\mathrm{d} p_{x 2}}{\mathrm{~d} \bar{e}_{A 1}}>0$ and $Q_{p_{x 2}}^{s}<0$ if $Q_{\bar{e}_{A 1}}^{s}>0$. 


\section{Derivation of (25)}

(B5) and (B6) jointly determine $\hat{p}_{x 2}$ and $\hat{p}_{e 1}$. These equations read in matrix notation

$$
\left[\begin{array}{cc}
\gamma_{e} & -\gamma_{x} \\
\left(\gamma_{p}-p_{e 1} e_{N 1} \eta_{N 1}\right) & -\gamma_{p}
\end{array}\right]\left[\begin{array}{l}
\hat{p}_{e 1} \\
\hat{p}_{x 2}
\end{array}\right]=\left[\begin{array}{c}
-\lambda_{A} \bar{e}_{A 1} \pi_{1} \hat{\bar{e}}_{A 1} \\
p_{e 1} \bar{e}_{A 1} \hat{\bar{e}}_{A 1}
\end{array}\right] .
$$

Solving the equation system (D1) by using Cramer's rule yields

$$
\frac{\hat{p}_{x 2}}{\hat{\bar{e}}_{A 1}}=\frac{\gamma_{e} p_{e 1} \bar{e}_{A 1}+\lambda_{A} \bar{e}_{A 1} \pi_{1}\left(\gamma_{p}-p_{e 1} e_{N 1} \eta_{N 1}\right)}{D}
$$

where $D:=-\gamma_{e} \gamma_{p}+\gamma_{x}\left(\gamma_{p}-p_{e 1} e_{N 1} \eta_{N 1}\right)$. To derive (25) we next prove

Lemma 1. If the utility function is homothetic, then

(i) $\lambda_{i}=\frac{p_{x 2}}{H\left(p_{x 2}\right)+p_{x 2}}=: \lambda$ for all $i=A, F, N$;

(ii) $\sigma_{i}=\frac{H_{p_{x 2}}}{H\left(p_{x 2}\right)} p_{x 2}=: \sigma$ for all $i=A, F, N$;

(iii) $\gamma_{x}=\gamma_{p}+\lambda \sigma\left(x_{A 1}^{s}+x_{N 1}^{s}\right)>0$;

(iv) $\gamma_{e}=\gamma_{p}>0$

(v) $D=\left(\gamma_{p}-p_{e 1} e_{N 1} \eta_{N 1}\right) \lambda \sigma\left(x_{A 1}^{s}+x_{N 1}^{s}\right)-\gamma_{p} p_{e 1} e_{N 1} \eta_{N 1}>0$.

\section{Proof:}

(i) Using $x_{i 1}=H\left(p_{x 2}\right) x_{i 2}$, which holds for homothetic utility functions, in $\lambda_{i}=\frac{p_{x 2} x_{i 2}}{x_{i 1}+p_{x 2} x_{i 2}}$ yields $\lambda_{i}=\frac{p_{x 2}}{H\left(p_{x 2}\right)+p_{x 2}}$.

(ii) Total differentiation of $x_{i 1}=H\left(p_{x 2}\right) x_{i 2}$ gives us $\hat{x}_{i 1}=\frac{H_{p_{x 2}}}{H\left(p_{x 2}\right)} p_{x 2} \hat{p}_{x 2}+\hat{x}_{i 2}$. Comparing this term with (A24) establishes $\sigma_{i}=\frac{H_{p_{x 2}}}{H\left(p_{x 2}\right)} p_{x 2}$.

(iii) Verify that

$$
\begin{aligned}
\gamma_{x} & =\gamma_{p}+\lambda_{F}\left(p_{x 2} x_{F 2}+x_{F 1} \sigma_{F}\right)-\sum_{j=A, N} \lambda_{j}\left(p_{x 2} \Delta x_{j 2}-x_{j 1} \sigma_{j}\right) \\
& =\gamma_{p}+\lambda \sum_{j=A, F, N}\left(p_{x 2} x_{j 2}+x_{j 1} \sigma\right)-\lambda \sum_{j=A, N} p_{x 2} x_{j 2}^{s} \\
& =\gamma_{p}+\lambda \sigma \sum_{j=A, F, N} x_{j 1}=\gamma_{p}+\lambda \sigma\left(x_{A 1}^{s}+x_{N 1}^{s}\right) .
\end{aligned}
$$

(iv) Making use of (A9), (A10) and (3) we obtain

$$
\begin{aligned}
\gamma_{e} & =\gamma_{p}+p_{x 2} x_{F 2}-\lambda \sum_{j=A, N} p_{e 1} e_{j} \\
& =\gamma_{p}+p_{x 2} x_{F 2}-\lambda\left[\Delta x_{A 1}+\Delta x_{N 1}+p_{x 2}\left(\Delta x_{A 2}+\Delta x_{N 2}\right)\right] \\
& =\gamma_{p}+p_{x 2} x_{F 2}-\lambda\left(x_{F 1}+p_{x 2} x_{F 2}\right)
\end{aligned}
$$


Inserting $\lambda=\frac{p_{x 2} x_{F 2}}{x_{F 1}+p_{x 2} x_{F 2}}$ in (D4) establishes $\gamma_{e}=\gamma_{p}$.

(v) follows from using Lemma 1 (iii) and 1 (iv) in the definition of $D$ and rearranging terms.

Taking advantage of Lemma 1(i)-(v) in (D2) establishes

$$
\frac{\hat{p}_{x 2}}{\hat{\bar{e}}_{A 1}}=\frac{\gamma_{p} \bar{e}_{A 1}\left(p_{e 1}+\lambda \pi_{1}\right)-\lambda \pi_{1} \bar{e}_{A 1} p_{e 1} e_{N 1} \eta_{N 1}}{\left(\gamma_{p}-p_{e 1} e_{N 1} \eta_{N 1}\right) \lambda \sigma\left(x_{A 1}^{s}+x_{N 1}^{s}\right)-\gamma_{p} p_{e 1} e_{N 1} \eta_{N 1}} .
$$

Finally, (D5) is inserted in (15b) to get, after some rearrangement of terms,

$$
\frac{\hat{e}_{F 1}}{\hat{\bar{e}}_{A 1}}=\frac{\bar{e}_{A 1} \gamma_{p} \lambda}{e_{F 1} D}\left(\sigma\left(x_{A 1}^{s}+x_{N 1}^{s}\right)+\pi_{1} e_{N 1} \eta_{N 1}\right) .
$$

\section{E. Proof of Proposition 3(ii)}

Total first-period profits are

$$
\Pi^{1}:=x_{A 1}^{s}+x_{N 1}^{s}-\left(p_{e 1}+\pi_{1}\right) \bar{e}_{A 1}-p_{e 1} e_{N 1},
$$

which are positive according to (10) and (11) (and footnote 7). Using that definition of $\Pi^{1}$ we rewrite $\gamma_{\theta 1}$ from $(28)$ as

$$
\gamma_{\theta 1}:=\frac{\pi_{1} e_{N 1}}{x_{A 1}^{s}+x_{N 1}^{s}}=\frac{\pi_{1} e_{N 1}}{\Pi^{1}+\pi_{1} \bar{e}_{A 1}+p_{e 1} e_{F 1}}=\frac{1}{\frac{\Pi^{1}}{\pi_{1} e_{N 1}}+\frac{\bar{e}_{A 1}}{e_{N 1}}\left(1+\frac{p_{e 1}}{\pi_{1}}\right)+\frac{p_{e 1}}{\pi_{1}}} .
$$

Obviously, $\gamma_{\theta 1}<1$ if $p_{e 1} \geq \pi_{1}$ and $\gamma_{\theta 1}<\frac{1}{3}$, if $p_{e 1} \geq \pi_{1}$ and $\bar{e}_{A 1} \geq e_{N 1}$.

\section{F. Emissions caps in both periods: The comparative static effects}

The competitive equilibrium of the model is characterized by (A1), (A2), (A3) for $i=N$, (A4) - (A12)

$$
\begin{aligned}
p_{x 2} X_{e_{A 2}}^{A}-p_{e 2}-\pi_{2} & =0 \\
e_{A 2}-\bar{e}_{A 2} & =0 .
\end{aligned}
$$

Total differentiation of these equations yields (A13), (A14), (A15) for $i=N$, (A16) - (A19), (A21) - (A24),

$$
\begin{array}{r}
p_{x 2} e_{A 2} X_{e_{A 2}}^{A} \hat{e}_{A 2}-p_{x 2} X_{e_{A 2}}^{A} \hat{p}_{x 2}-p_{e 2} \hat{p}_{e 2}-\pi_{2} \hat{\pi}_{2}=0 \\
\hat{e}_{A 2}-\hat{\bar{e}}_{A 2}=0 \\
\left(X_{e_{A 1}}^{A}-p_{e 1}\right) e_{A 1} \hat{e}_{A 1}+\left(p_{x 2} X_{e_{A 2}}^{A}-p_{e 2}\right) e_{A 2} \hat{e}_{A 2}-x_{A 1} \hat{x}_{A 1}-p_{x 2} x_{A 2} \hat{x}_{A 2} \\
-\left(e_{A 1}+e_{A 2}\right) p_{e 1} \hat{p}_{e 1}+\left[X^{A}\left(e_{A 2}\right)-x_{A 2}\right] p_{x 2} \hat{p}_{x 2}=0 .
\end{array}
$$


Making use of (A24) in (F5), (A21) and (A22) yields

$$
\hat{x}_{A 2}=\frac{p_{x 2} \Delta x_{A 2}-x_{A 1} \sigma_{A}}{y_{A}} \hat{p}_{x 2}-\frac{p_{e 1} e_{A}}{y_{A}} \hat{p}_{e 1}+\frac{\pi_{1} \bar{e}_{A 1}}{y_{A}} \hat{\bar{e}}_{A 1}+\frac{\pi_{2} \bar{e}_{A 2}}{y_{A}} \hat{\bar{e}}_{A 2},
$$

(B2) and (B3).

Next, we insert (A15) and (A16) and (F4) in (A23) to obtain

$$
\tilde{\gamma}_{p}\left(\hat{p}_{x 2}-\hat{p}_{e 1}\right)+\left(p_{e 2}+\pi_{2}\right) \bar{e}_{A 2} \hat{\bar{e}}_{A 2}=p_{x 2} x_{A 2} \hat{x}_{A 2}+p_{x 2} x_{B 2} \hat{x}_{B 2}+p_{x 2} x_{F 2} \hat{x}_{F 2},
$$

where $\tilde{\gamma}_{p}:=-e_{N 2} \eta_{N 2} p_{e 2}>0$.

Inserting (F6), (B2), (B3) in (F7) and rearranging terms we get

$$
\tilde{\gamma}_{x} \hat{p}_{x 2}-\tilde{\gamma}_{e} \hat{p}_{e 1}=\lambda_{A} \pi_{1} \bar{e}_{A 1} \hat{\bar{e}}_{A 1}+\left[\lambda_{A} \pi_{2}-\left(p_{e 2}+\pi_{2}\right)\right] e_{A 2} \hat{\bar{e}}_{A 2}
$$

where

$$
\begin{aligned}
\tilde{\gamma}_{x} & :=\tilde{\gamma}_{p}+\lambda_{F}\left(p_{x 2} x_{F 2}+x_{F 1} \sigma_{F}\right)-\sum_{j=A, N} \lambda_{j}\left(p_{x 2} \Delta x_{j 2}-x_{j 1} \sigma_{j}\right), \\
\tilde{\gamma}_{e} & :=\tilde{\gamma}_{p}+p_{x 2} x_{F 2}-\sum_{j=A, N} \lambda_{j} p_{e 1} e_{j} .
\end{aligned}
$$

Solving (A18) with respect to $\hat{e}_{F t}$, inserting this term in (A19) and making use of (A14) (A17) and (F2) we obtain

$$
-\left(\tilde{\gamma}_{p}-p_{e 1} e_{N 1} \eta_{N 1}\right) \hat{p}_{e 1}+\tilde{\gamma}_{p} \hat{p}_{x 2}=-p_{e 1} \bar{e}_{A 1} \hat{\bar{e}}_{A 1}-p_{e 2} \bar{e}_{A 2} \hat{\bar{e}}_{A 2}
$$

Inserting (A14) and (A17) in (A18) for $t=1$ we get

$$
\hat{e}_{F 1} e_{F 1}=\bar{e}_{A 1} \hat{\bar{e}}_{A 1}+e_{N 1} \eta_{N 1} \hat{p}_{e 1} \text {. }
$$

Solving (F9) for $\hat{p}_{e 1}$ and insert this term into (F10) establishes

$$
\hat{e}_{F 1} e_{F 1}=\bar{e}_{A 1} \hat{\bar{e}}_{A 1}+\frac{e_{N 1} \eta_{N 1} p_{e 1} \bar{e}_{A 1}}{\tilde{\gamma}_{p}-p_{e 1} e_{N 1} \eta_{N 1}} \hat{\bar{e}}_{A 1}+\frac{e_{N 1} \eta_{N 1} p_{e 1} \bar{e}_{A 2}}{\tilde{\gamma}_{p}-p_{e 1} e_{N 1} \eta_{N 1}} \hat{\bar{e}}_{A 2}+\frac{\tilde{\gamma}_{p} e_{N 1} \eta_{N 1}}{\tilde{\gamma}_{p}-p_{e 1} e_{N 1} \eta_{N 1}} \hat{p}_{x 2}
$$

Next, solving (F8) and (F9) yields

$$
\begin{aligned}
\frac{\hat{p}_{x 2}}{\hat{\bar{e}}_{A 1}} & =\frac{\tilde{\gamma}_{p} \bar{e}_{A 1}\left(p_{e 1}+\lambda \pi_{1}\right)-\lambda \pi_{1} \bar{e}_{A 1} p_{e 1} e_{N 1} \eta_{N 1}}{\tilde{D}} \\
\frac{\hat{p}_{x 2}}{\hat{\bar{e}}_{A 2}} & =-\frac{(1-\lambda) \bar{e}_{A 2} \pi_{2}\left(\tilde{\gamma}_{p}-p_{e 1} e_{N 1} \eta_{N 1}\right)-p_{e 1}^{2} \bar{e}_{A 2} e_{N 1} \eta_{N 1}}{\tilde{D}}
\end{aligned}
$$

where $\tilde{D}:=-\tilde{\gamma}_{e} \tilde{\gamma}_{p}+\tilde{\gamma}_{x}\left(\tilde{\gamma}_{p}-p_{e 1} e_{N 1} \eta_{N 1}\right)$. Using the same arguments as in Lemma 1 one can show that

$$
\tilde{D}=\left(\tilde{\gamma}_{p}-p_{e 1} e_{N 1} \eta_{N 1}\right) \lambda \sigma\left(\sum_{j=A, F, N} x_{j 1}\right)-\tilde{\gamma}_{p} p_{e 1} e_{N 1} \eta_{N 1}>0
$$


Inserting (F12) and (F13), respectively, in (F11) we obtain

$$
\begin{aligned}
\frac{\hat{e}_{F 1}}{\hat{\bar{e}}_{A 1}} & =\frac{\tilde{\gamma}_{p} \bar{e}_{A 1} \lambda}{e_{F 1} \tilde{D}}\left(\sigma \sum_{j=A, F, N} x_{j 1}+\pi_{1} e_{N 1} \eta_{N 1}\right) \\
\frac{\hat{e}_{F 1}}{\hat{\bar{e}}_{A 2}} & =\frac{p_{e 1} e_{N 1} \eta_{N 1} \bar{e}_{A 2}}{e_{F 1} \tilde{D}}\left(\lambda \sigma \sum_{j=A, F, N} x_{j 1}+(1-\lambda) \pi_{2} e_{N 2} \eta_{N 2}\right) .
\end{aligned}
$$

Finally, we rearrange $(\mathrm{F} 15)$ with the help of $\lambda x_{j 1}=(1-\lambda) p_{x 2} x_{j 2}$ to

$$
\frac{\hat{e}_{F 1}}{\hat{\bar{e}}_{A 2}}=\frac{(1-\lambda) p_{e 1} e_{N 1} \eta_{N 1} \bar{e}_{A 2}}{e_{F 1} \tilde{D}}\left(\sigma \sum_{j=A, F, N} p_{x 2} x_{j 2}+\pi_{2} e_{N 2} \eta_{N 2}\right) \text {. }
$$

\section{G. Proof of Proposition 5}

Follows from verifying that (F11) with $\hat{\bar{e}}_{A 2}=0,(\mathrm{~F} 12),(\mathrm{F} 14)$ are qualitatively equal to (15b), (D5) and (D6). The only difference is that $\gamma_{p}:=-p_{e 1}\left(e_{A 2} \eta_{A 2}+e_{N 2} \eta_{N 1}\right)>0$ in (15b), (D5) and (D6) is replaced by $\tilde{\gamma}_{p}:=-p_{e 2} e_{N 2} \eta_{N 2}>0$ in (F11), (F12), (F14).

\section{H. Proof of Proposition 6}

Contrary to the ascertion suppose that $\hat{p}_{x 2} / \hat{\bar{e}}_{A 2}>0$. In that case we obtain $\hat{e}_{F 1} / \hat{\bar{e}}_{A 2}>0$ and $\hat{e}_{F 1} / \hat{\bar{e}}_{A 2}<0$. Using the same arguments as in the proof of Proposition 1 the last inequalities translate into $\left(\hat{x}_{A 1}^{s} x_{A 1}^{s}+\hat{x}_{N 1}^{s} x_{N 1}^{s}\right) / \hat{\bar{e}}_{A 2}<0$ and $\left(\hat{x}_{A 2}^{s} x_{A 2}^{s}+\hat{x}_{N 2}^{s} x_{N 2}^{s}\right) / \hat{\bar{e}}_{A 2}>0$ or $\hat{q}^{s} / \hat{\bar{e}}_{A 2}<0$. On the demand side we get $\hat{q}^{d} / \hat{\bar{e}}_{A 2}=\sigma \cdot\left(\hat{p}_{x 2} / \hat{\bar{e}}_{A 2}\right)>0$ for $\hat{p}_{x 2} / \hat{\bar{e}}_{A 2}>0$ which implies $\left(\hat{q}^{s}-\hat{q}^{d}\right) / \hat{\bar{e}}_{A 2}<0$. This condition contradicts the necessary equilibrium condition $\left(\hat{q}^{s}-\hat{q}^{d}\right) / \hat{\bar{e}}_{A 2}=0$, which proves $\hat{p}_{x 2} / \hat{\bar{e}}_{A 2}<0 . \hat{p}_{e 1} / \hat{\bar{e}}_{A 2}<0$ follows from $\hat{p}_{x 2} / \hat{\bar{e}}_{A 2}<0$ and (F9), and $\mathrm{d} e_{F 1} / \mathrm{d} \bar{e}_{A 2}>-1$ follows from (F11). 